\title{
Basis-free Solution to General Linear Quater- nionic Equation
}

\author{
Changpeng Shao, Hongbo Li, Lei Huang
}

\begin{abstract}
A linear quaternionic equation in one quaternionic variable $\mathbf{q}$ is of the form $\mathbf{a}_{1} \mathbf{q} \mathbf{b}_{1}+\mathbf{a}_{2} \mathbf{q} \mathbf{b}_{2}+\cdots+\mathbf{a}_{m} \mathbf{q} \mathbf{b}_{m}=\mathbf{c}$, where the $\mathbf{a}_{i}, \mathbf{b}_{j}, \mathbf{c}$ are given quaternionic coefficients. If introducing basis elements $\mathbf{i}, \mathbf{j}, \mathbf{k}$ of pure quaternions, then the quaternionic equation becomes four linear equations in four unknowns over the reals, and solving such equations is trivial. On the other hand, finding a quaternionic rational function expression of the solution that involves only the input quaternionic coefficients and their conjugates, called a basis-free solution, is non-trivial.

In 1884, Sylvester initiated the study of basis-free solution to linear quaternionic equation. He considered the three-termed equation aq + $\mathbf{q} \mathbf{b}=\mathbf{c}$, and found its solution $\mathbf{q}=\left(\mathbf{a}^{2}+\mathbf{b} \overline{\mathbf{b}}+\mathbf{a}(\mathbf{b}+\overline{\mathbf{b}})\right)^{-1}(\mathbf{a c}+\mathbf{c} \overline{\mathbf{b}})$ by successive left and right multiplications. In 2013, Schwartz extended the technique to the four-termed equation, and obtained the basis-free solution in explicit form.

This paper solves the general problem for arbitrary number of terms in the non-degenerate case.
\end{abstract}

Mathematics Subject Classification (2010). Primary 11R52; Secondary 15A66, 16Z05.

Keywords. Linear quaternionic equation; Sylvester equation; Basis-free solution; Clifford algebra.

\section{Introduction}

A quaternionic variable is $\mathbf{q}:=u+x \mathbf{i}+y \mathbf{j}+z \mathbf{k}$, where the $u, x, y, z$ are realvalued variables, and $1, \mathbf{i}, \mathbf{j}, \mathbf{k}$ are the fixed basis of quaternions. A quaternionic monomial of degree $m$ is of the form $\mathbf{a}_{1} \mathbf{q} \mathbf{a}_{2} \mathbf{q} \cdots \mathbf{a}_{m} \mathbf{q} \mathbf{a}_{m+1}$, where the quaternionic variable $\mathbf{q}$ occurs $m$ times, and the $\mathbf{a}_{l}$ are quaternionic coefficients.

By rewriting the quaternionic variable and the quaternionic coefficients as linear combinations of the fixed basis, a quaternionic polynomial equation is converted into four real polynomial equations in four real variables, and 
methods of real polynomial system solving can be applied to find solutions for the $u, x, y, z$, then for the unknown $\mathbf{q}$. So if the basis elements $\mathbf{i}, \mathbf{j}, \mathbf{k}$ are introduced, then solving quaternionic equations is reduced to solving real polynomial equations.

For example, for a linear quaternionic equations of $n$ terms [16], i.e., an equation of the form

$$
\mathbf{c}_{1} \mathbf{q} \mathbf{b}_{1}+\mathbf{c}_{2} \mathbf{q} \mathbf{b}_{2}+\cdots+\mathbf{c}_{n-1} \mathbf{q} \mathbf{b}_{n-1}=\mathbf{d},
$$

by introducing real-valued variables $u, x, y, z$ for quaternionic variable $\mathbf{q}$, and real-valued coordinates for the $\mathbf{c}_{i}, \mathbf{b}_{j}, \mathbf{d}$, (1.1) is converted into four real linear equations in four unknowns, called the associated real linear system of the equation. Standard methods such as Gaussian elimination can be used to solve the linear system.

For an input quaternionic equation where the basis elements $\mathbf{i}, \mathbf{j}, \mathbf{k}$ do not occur explicitly in the coefficients, to keep the equation solving within the framework of quaternions instead of real numbers, it is sometimes desired that the quaternionic solution be expressed as a quaternionic rational function in the input quaternionic coefficients and their conjugates only, so that if the input equation does not involve the basis elements $\mathbf{i}, \mathbf{j}, \mathbf{k}$, nor does the solution. Such a solution, if exists, is called a basis-free solution.

For example, for the linear quaternionic equation (1.1), if $n=2$, then solving $\mathbf{c q b}=\mathbf{d}$ is trivial, and the basis-free solution is $\mathbf{q}=\mathbf{c}^{-1} \mathbf{d} \mathbf{b}^{-1}$ under the non-degeneracy condition $\mathbf{c b} \neq 0$. If $n=3$, by multiplying both sides of (1.1) from the right with $\mathbf{b}_{1}^{-1}$ and from the left with $\mathbf{c}_{2}^{-1}$, we get the following Sylvester's equation [20]:

$$
\mathbf{s q}+\mathbf{q t}=\mathbf{u}
$$

where $\mathbf{s}=\mathbf{c}_{2}^{-1} \mathbf{c}_{1}, \mathbf{t}=\mathbf{b}_{2} \mathbf{b}_{1}^{-1}$, and $\mathbf{u}=\mathbf{c}_{2}^{-1} \mathbf{d b}_{1}^{-1}$ under the non-degeneracy condition $\mathbf{c}_{2} \mathbf{b}_{1} \neq 0$. It is well known that the basis-free solution of (1.2) can be obtained as follows:

1. Multiply both sides of (1.2) from the left with s:

$$
\mathbf{s}^{2} \mathbf{q}+\mathbf{s q t}=\mathbf{s u} .
$$

2. Multiply both sides of (1.2) from the right with $\overline{\mathbf{t}}$ :

$$
\mathbf{s q} \overline{\mathbf{t}}+\mathbf{t} \overline{\mathbf{t} q}=\mathbf{u} \overline{\mathrm{t}}
$$

3. Add up (1.3) and (1.4):

$$
\left(\mathbf{s}^{2}+\mathbf{t} \overline{\mathbf{t}}+(\mathbf{t}+\overline{\mathbf{t}}) \mathbf{s}\right) \mathbf{q}=\mathbf{s u}+\mathbf{u} \overline{\mathbf{t}}
$$

4. The solution is

$$
\mathbf{q}=\left(\mathbf{s}^{2}+\mathbf{t} \overline{\mathbf{t}}+(\mathbf{t}+\overline{\mathbf{t}}) \mathbf{s}\right)^{-1}(\mathbf{s u}+\mathbf{u} \overline{\mathbf{t}})
$$

under the non-degeneracy condition $\mathbf{s}^{2}+\mathbf{t} \overline{\mathbf{t}}+(\mathbf{t}+\overline{\mathbf{t}}) \mathbf{s} \neq 0$.

Notation. Following [1], for arbitrary quaternions $\mathbf{u}, \mathbf{v}$, we use "( $\mathbf{u} \mid \mathbf{v})$ " to denote the following linear operator upon quaternions:

$$
(\mathbf{u} \mid \mathbf{v}) \mathbf{q}:=\mathbf{u q v}, \text { for any } \mathbf{q} \in \mathbb{H} \text {. }
$$


This operator satisfies the following composition rule:

$$
\left(\mathbf{u}_{1} \mid \mathbf{v}_{1}\right)\left(\mathbf{u}_{2} \mid \mathbf{v}_{2}\right)=\left(\mathbf{u}_{1} \mathbf{u}_{2} \mid \mathbf{v}_{2} \mathbf{v}_{1}\right) .
$$

By the above notation, the procedure of solving (1.2) can be written succinctly as the following identity:

$$
((\mathbf{s} \mid 1)+(1 \mid \overline{\mathbf{t}}))((\mathbf{s} \mid 1)+(1 \mid \mathbf{t}))=\left(\mathbf{s}^{2}+\mathbf{t} \overline{\mathbf{t}}+(\mathbf{t}+\overline{\mathbf{t}}) \mathbf{s} \mid 1\right) .
$$

In the literature, [10] extended Sylvester's equation to algebraic division ring, and [5, 15] classified the solutions of Sylvester's equation in various degenerate cases. For the more general linear quaternionic equation (1.1), 7. 8, 18 investigated the non-degeneracy conditions of the equation by considering its associated real linear system. When the number of terms $n=4$, [19] obtained the basis-free solution in explicit form in the non-degenerate case, by reducing the number of terms to three with successive left and right multiplications.

There are also many results on properties of nonlinear quaternionic equations and algorithms to solve them [2, 6, 9, 11, 13, 14, 17, 21, These results, concerning only the derived real polynomial system of the input equations, do not lead to basis-free solution. Even in the simplest case of linear quaternionic equation (1.1) whose associated real linear system is nondegenerate, finding the basis-free solution is highly non-trivial, and there is still no result for $n>4$ in the literature.

In [19], the following idea is proposed to obtain a non basis-free solution of (1.1). Introduce the coordinate form of the quaternionic coefficients $\mathbf{b}_{p}$ for $p=1 . . n-1$ :

$$
\mathbf{b}_{p}=b_{p 0}+b_{p 1} \mathbf{i}+b_{p 2} \mathbf{j}+b_{p 3} \mathbf{k} .
$$

Substituting them into (1.1) and expanding the result, we get

$$
\mathbf{a}_{0} \mathbf{q}+\mathbf{a}_{1} \mathbf{q} \mathbf{i}+\mathbf{a}_{2} \mathbf{q} \mathbf{j}+\mathbf{a}_{3} \mathbf{q} \mathbf{k}=\mathbf{d},
$$

where for $i=0 . .3$,

$$
\mathbf{a}_{i}=\sum_{p=1}^{n-1} b_{p i} \mathbf{c}_{p} \in \mathbb{H} .
$$

(1.11) is called the revised starting form [19] of equation (1.1). Non basisfree solution in explicit form can be computed by successive left and right multiplications, or simply by Gaussian elimination.

To find the basis-free solution of (1.1), our first approach is first to derive the explicit form of the non basis-free solution of (1.1) by solving (1.11), then to convert it into a basis-free quaternionic expression. This procedure is difficult, but we manage to make it by brute-force try and error. The outline of this approach is as follows.

Let $\mathbf{f}$ be the $\mathbb{R}$-linear isomorphism from $\mathbb{H}$ to $\mathbb{R}^{4}$ mapping $1, \mathbf{i}, \mathbf{j}, \mathbf{k}$ to the orthonormal basis $\mathbf{e}_{0}, \mathbf{e}_{1}, \mathbf{e}_{2}, \mathbf{e}_{3}$ of $\mathbb{R}^{4}$. The associated real linear system of (1.11) is of the form $\mathbf{A f}(\mathbf{q})=\mathbf{f}(\mathbf{d})$, where $\mathbf{A}$ is a $4 \times 4$ real matrix whose entries are polynomials in the coordinates of the $\mathbf{a}_{l}$ for $l=0 . .3$. Under the nondegeneracy condition $\operatorname{det}(\mathbf{A}) \neq 0$, the solution to (1.11) is $\mathbf{f}(\mathbf{q})=\mathbf{A}^{-1} \mathbf{f}(\mathbf{d})=$ 
$\operatorname{adj}(\mathbf{A}) \mathbf{f}(\mathbf{d}) / \operatorname{det}(\mathbf{A})$, where $\operatorname{adj}(\mathbf{A})$ is the adjugate matrix of $\mathbf{A}$. The result is a basis-dependent vector-valued rational function in the coordinates of the $\mathbf{a}_{l}$ and $\mathbf{d}$, where each numerator is a 1984-termed polynomial, and the denominator $\operatorname{det}(\mathbf{A})$ is a 196 -termed polynomial.

By setting up a list of correspondences between several Clifford algebraic expressions over $\mathbb{R}^{4}$ and their basis-free preimages in $\mathbb{H}$ under $\mathbf{f}$, we manage to rewrite $\operatorname{adj}(\mathbf{A})$ as the matrix form of the following linear operator acting upon $\mathbb{H}:\left(\mathbf{p}_{0} \mid 1\right)+\left(\mathbf{p}_{1} \mid \mathbf{i}\right)+\left(\mathbf{p}_{2} \mid \mathbf{j}\right)+\left(\mathbf{p}_{3} \mid \mathbf{k}\right)$, where each $\mathbf{p}_{l}$ is a basis-free quaternionic polynomial in the $\mathbf{a}_{l}$ and their conjugates. Similarly, we rewrite $\operatorname{det}(\mathbf{A})$ as a basis-free quaternionic polynomial in the $\mathbf{a}_{l}$ and their conjugates.

Then by substituting the expressions (1.12) of the $\mathbf{a}_{l}$ into the above result, after some hard manipulations upon the expanded summations, we get the basis-free quaternionic solution to (1.1). As a byproduct, we get a procedure of left and right multiplications by expressions of the coefficients of (1.1), so that the basis-free solution is obtained by adding up the results of the multiplications, similar to the procedure from (1.3) to (1.6) in solving Sylvester's equation.

The first approach is not only complicated, but also unable to interpret why some Clifford algebraic expressions over $\mathbb{R}^{4}$ occur naturally in the solution, and why they can significantly reduce the size of the solution expression.

Our second approach is first to embed the algebra of quaternions into $C L\left(\mathbb{R}^{4}\right)$, so that the input equation becomes a linear Clifford algebraic equation, then to solve the linear Clifford algebraic equation, and then to project the solution onto $\mathbb{H}$ to get the basis-free solution of the input quaternionic equation. It turns out that this approach is much easier, and very elegant.

There are various embeddings of $\mathbb{H}$ as a subalgebra into $C L\left(\mathbb{R}^{4}\right)$. A classical algebraic homomorphism 4 is generated by the correspondence between the basis elements $1 \leftrightarrow 1,-\mathbf{e}_{23} \leftrightarrow \mathbf{i}, \mathbf{e}_{13} \leftrightarrow \mathbf{j}$, and $-\mathbf{e}_{12} \leftrightarrow \mathbf{k}$. To include both this algebraic homomorphism and the linear isomorphism $\mathbf{f}: \mathbb{H} \longrightarrow \mathbb{R}^{4}$ within the same framework, we need a linear map $\pi$ from $C L\left(\mathbb{R}^{4}\right)$ to $\mathbb{H}$, so that $\pi$ restricted to $\mathbb{R}^{4}$ is just $\mathbf{f}^{-1}$, while $\pi$ restricted to the subalgebra $\left\langle 1, \mathbf{e}_{12}, \mathbf{e}_{13}, \mathbf{e}_{23}\right\rangle_{\mathbb{R}}$ gives the classical algebraic homomorphism. Such a linear map can be easily constructed, and it "lift"s the input quaternionic equation (1.1) to the following linear Clifford algebraic equation:

$$
\sum_{r=1}^{n-1} \mathbf{f}\left(\mathbf{c}_{r}\right) \mathbf{f}(\overline{\mathbf{q}}) \mathbf{f}\left(\mathbf{b}_{r}\right)\left(1+\mathbf{e}_{0123}\right)=\mathbf{f}(\mathbf{d})\left(1+\mathbf{e}_{0123}\right) .
$$

Solving this equation can be done easily by hand. The solution when projected to $\mathbb{H}$ by $\pi$ gives the solution that is obtained by the first approach in exactly the same form.

Moreover, once equation (1.1) is solved, then a more general linear quaternionic equation involving one quaternionic variable and its conjugate can be solved readily to obtain a basis-free solution.

So this paper solves the long-lasting problem of solving the linear quaternionic equation of arbitrary number of terms for the basis-free solution in 
explicit form, by a procedure of successive left and right multiplications with the input quaternionic coefficients and their conjugates, in the non-degenerate case. The content is arranged as follows. Section 2 introduces some notations and basics of linear quaternionic equations. Section 3 establishes the correspondence between some typical Clifford algebraic expressions over $\mathbb{R}^{4}$ and their basis-free quaternionic representations. Section 4 presents the associated real linear system approach. Section 5 presents the Clifford algebra approach. Section 6 solves the general linear quaternionic equation with conjugate in the non-degenerate case.

\section{Quaternions and linear quaternionic equations}

In a quaternionic variable $\mathbf{q}:=u+x \mathbf{i}+y \mathbf{j}+z \mathbf{k}, u$ is called the scalar part (or real part), and $x \mathbf{i}+y \mathbf{j}+z \mathbf{k}$ is called the vector part (or pure imaginary part). The quaternionic conjugate is denoted by the over-bar symbol, while the $\mathbb{R}$-linear operators extracting the real part and the pure imaginary part are denoted by "Re" and "Im" respectively:

$$
\overline{\mathbf{q}}=u-x \mathbf{i}-y \mathbf{j}-z \mathbf{k}, \quad \operatorname{Re}(\mathbf{q})=\frac{\mathbf{q}+\overline{\mathbf{q}}}{2}, \quad \operatorname{Im}(\mathbf{q})=\frac{\mathbf{q}-\overline{\mathbf{q}}}{2} .
$$

Obviously $\mathbf{q} \overline{\mathbf{q}}=\overline{\mathbf{q}} \mathbf{q}=u^{2}+x^{2}+y^{2}+z^{2}$, and $\mathbf{q}+\overline{\mathbf{q}}=2 u$; they are both real-valued.

Let $\mathbf{e}_{0}, \mathbf{e}_{1}, \mathbf{e}_{2}, \mathbf{e}_{3}$ be a fixed basis of $\mathbb{R}^{4}$. Define a linear isomorphism from $\mathbb{H}$ to $\mathbb{R}^{4}$ as following:

$$
\mathbf{f}: \quad 1 \mapsto \mathbf{e}_{0}, \quad \mathbf{i} \mapsto \mathbf{e}_{1}, \quad \mathbf{j} \mapsto \mathbf{e}_{2}, \quad \mathbf{k} \mapsto \mathbf{e}_{3} .
$$

For any $\mathbf{a} \in \mathbb{H}$, denote

$$
\mathbf{a}:=\mathbf{f}(\mathbf{a}), \quad \overline{\mathbf{a}}:=\mathbf{f}(\overline{\mathbf{a}}) .
$$

Under the above linear isomorphism between $\mathbb{H}$ and $\mathbb{R}^{4}$, the left (or right) multiplication by $\mathbf{p}=p_{0}+p_{1} \mathbf{i}+p_{2} \mathbf{j}+p_{3} \mathbf{k} \in \mathbb{H}$ can be taken as a linear transformation in $\mathbb{R}^{4}$. The two multiplications have the following $4 \times 4$ real matrix form:

$$
(\mathbf{p} \mid 1)=\left(\begin{array}{rrrr}
p_{0} & -p_{1} & -p_{2} & -p_{3} \\
p_{1} & p_{0} & -p_{3} & p_{2} \\
p_{2} & p_{3} & p_{0} & -p_{1} \\
p_{3} & -p_{2} & p_{1} & p_{0}
\end{array}\right), \quad(1 \mid \mathbf{p})=\left(\begin{array}{rrrr}
p_{0} & -p_{1} & -p_{2} & -p_{3} \\
p_{1} & p_{0} & p_{3} & -p_{2} \\
p_{2} & -p_{3} & p_{0} & p_{1} \\
p_{3} & p_{2} & -p_{1} & p_{0}
\end{array}\right) .
$$

They have the following simple properties:

$$
\begin{array}{ll}
\text { (i). } & (\overline{\mathbf{p}} \mid 1)=(\mathbf{p} \mid 1)^{T} ; \\
\text { (ii). } & (1 \mid \overline{\mathbf{p}})=(1 \mid \mathbf{p})^{T} ; \\
\text { (iii). } & \operatorname{det}(\mathbf{p} \mid 1)=\operatorname{det}(1 \mid \mathbf{p})=(\mathbf{p} \overline{\mathbf{p}})^{2} .
\end{array}
$$

For any unit quaternion $\mathbf{p}$, both $(\mathbf{p} \mid 1)$ and $(1 \mid \mathbf{p})$ are in $S O(4)$. Conversely, any element $\mathbf{M} \in S O(4)$ is of the form $\mathbf{M}=(\mathbf{p} \mid 1)(1 \mid \mathbf{q})$ for two unit quaternions $\mathbf{p}, \mathbf{q}[3]$. 
Consider the revised starting form (1.11). In matrix form,

$$
\begin{aligned}
\mathbf{A}:= & \left(\mathbf{a}_{0} \mid 1\right)+\left(\mathbf{a}_{1} \mid \mathbf{i}\right)+\left(\mathbf{a}_{2} \mid \mathbf{j}\right)+\left(\mathbf{a}_{3} \mid \mathbf{k}\right) \\
= & \left(\begin{array}{rr}
a_{00}-a_{11}-a_{22}-a_{33} & -a_{01}-a_{10}-a_{23}+a_{32} \\
a_{01}+a_{10}-a_{23}+a_{32} & a_{00}-a_{11}+a_{22}+a_{33} \\
a_{02}+a_{13}+a_{20}-a_{31} & a_{03}-a_{12}-a_{21}-a_{30} \\
a_{03}-a_{12}+a_{21}+a_{30} & -a_{02}-a_{13}+a_{20}-a_{31} \\
-a_{02}+a_{13}-a_{20}-a_{31} & -a_{03}-a_{12}+a_{21}-a_{30} \\
-a_{03}-a_{12}-a_{21}+a_{30} & a_{02}-a_{13}-a_{20}-a_{31} \\
a_{00}+a_{11}-a_{22}+a_{33} & -a_{01}+a_{10}-a_{23}-a_{32} \\
a_{01}-a_{10}-a_{23}-a_{32} & a_{00}+a_{11}+a_{22}-a_{33}
\end{array}\right) .
\end{aligned}
$$

Proposition 2.1. For any $4 \times 4$ real matrix $\mathbf{M}$, there exist $\mathbf{p}_{l} \in \mathbb{H}$ for $l=0 . .3$ such that

$$
\mathbf{M}=\left(\mathbf{p}_{0} \mid 1\right)+\left(\mathbf{p}_{1} \mid \mathbf{i}\right)+\left(\mathbf{p}_{2} \mid \mathbf{j}\right)+\left(\mathbf{p}_{3} \mid \mathbf{k}\right) .
$$

More explicitly, if $\mathbf{M}=\left(m_{i j}\right)_{i, j=0 . .3}$, then

$$
\begin{aligned}
4 \mathbf{p}_{0}= & \left(m_{00}+m_{11}+m_{22}+m_{33}\right)-\left(m_{01}-m_{10}+m_{23}-m_{32}\right) \mathbf{i} \\
& -\left(m_{02}-m_{13}-m_{20}+m_{31}\right) \mathbf{j}-\left(m_{03}+m_{12}-m_{21}-m_{30}\right) \mathbf{k}, \\
4 \mathbf{p}_{1}= & -\left(m_{01}-m_{10}-m_{23}+m_{32}\right)-\left(m_{00}+m_{11}-m_{22}-m_{33}\right) \mathbf{i} \\
& -\left(m_{03}+m_{12}+m_{21}+m_{30}\right) \mathbf{j}+\left(m_{02}-m_{13}+m_{20}-m_{31}\right) \mathbf{k}, \\
4 \mathbf{p}_{2}= & -\left(m_{02}+m_{13}-m_{20}-m_{13}\right)+\left(m_{03}-m_{12}-m_{21}+m_{30}\right) \mathbf{i} \\
& -\left(m_{00}-m_{11}+m_{22}-m_{33}\right) \mathbf{j}-\left(m_{01}+m_{10}+m_{23}+m_{32}\right) \mathbf{k}, \\
4 \mathbf{p}_{3}= & -\left(m_{03}-m_{12}+m_{21}-m_{30}\right)-\left(m_{02}+m_{13}+m_{20}+m_{31}\right) \mathbf{i} \\
& +\left(m_{01}+m_{10}-m_{23}-m_{32}\right) \mathbf{j}-\left(m_{00}-m_{11}-m_{22}+m_{33}\right) \mathbf{k} .
\end{aligned}
$$

As a corollary, any 4 real linear equations in 4 unknowns can be written as a linear quaternionic equation.

Proof. We need to solve the equation $\mathbf{A}=\mathbf{M}$ for the unknowns $a_{i j}$ from the expression of $\mathbf{A}$ in (2.6). The entries of $\mathbf{A}$ can be classified into four groups: (i) the four diagonal elements, as they are linear expressions in $a_{00}, a_{11}, a_{22}, a_{33}$; (ii) the four anti-diagonal elements (those with row and column numbers $(1,4),(2,3),(3,2),(4,1))$, as they are linear expressions in $a_{03}, a_{30}, a_{12}, a_{21}$; (iii) the four elements with row and column numbers $(1,2),(2,1),(3,4)$, $(4,3)$, as they are linear expressions in $a_{01}, a_{10}, a_{23}, a_{32}$; (iv) the four elements with row and column numbers $(1,3),(3,1),(2,4),(4,2)$, as they are linear expressions in $a_{02}, a_{20}, a_{13}, a_{31}$.

First consider group (i). The four equalities of the four corresponding elements in $\mathbf{A}$ and $\mathbf{M}$ respectively can be written as

$$
\left(\begin{array}{rrrr}
1 & -1 & -1 & -1 \\
1 & -1 & 1 & 1 \\
1 & 1 & -1 & 1 \\
1 & 1 & 1 & -1
\end{array}\right)\left(\begin{array}{l}
a_{00} \\
a_{11} \\
a_{22} \\
a_{33}
\end{array}\right)=\left(\begin{array}{l}
m_{00} \\
m_{11} \\
m_{22} \\
m_{33}
\end{array}\right)
$$


The determinant of the coefficient matrix is nonzero, so the equations have a unique solution for the unknowns $a_{00}, a_{11}, a_{22}, a_{33}$.

Similarly, in each of the other three groups, the four equalities of the four corresponding elements in $\mathbf{A}$ and $\mathbf{M}$ respectively form a non-degenerate linear system, so the solutions are unique. Solving all the linear equations, we get the solution (2.8).

\section{Quaternionic representations of some Clifford algebraic expressions over $\mathbb{R}^{4}$}

The Clifford algebra over $\mathbb{R}^{4}$ is the quotient of the tensor algebra generated by $\mathbb{R}^{4}$ modulo the two-sided ideal generated by elements of the form $\mathbf{x} \otimes \mathbf{x}-\mathbf{x} \cdot \mathbf{x}$ for all vectors $\mathbf{x} \in \mathbb{R}^{4}$, where the dot symbol denotes the inner product in $\mathbb{R}^{4}$. The associative product induced by the quotient from the tensor product is called the Clifford product, denoted by the juxtaposition of elements. The antisymmetrization of the Clifford product is called the exterior product, also known as the outer product, and is denoted by the wedge symbol.

Clifford algebra $C L\left(\mathbb{R}^{4}\right)$ is $\mathbb{Z}_{2}$-graded. The Clifford product of even number of vectors is called an even element, so is any linear combination of even elements. Similarly, the Clifford product of odd number of vectors is called an odd element, so is any linear combination of odd elements. The Clifford algebra is also $\mathbb{Z}_{5}$-graded, with grades ranging from 0 to 4 : a 0 -graded element is a scalar, a 1-graded element is a vector, and an $r$-graded element for $r>1$ is the linear combination of outer products of $r$ vectors. The outer product of vectors in $\mathbb{R}^{4}$ whose number is $>4$ is zero. A general element $\mathbf{A}$ of $C L\left(\mathbb{R}^{4}\right)$ is a linear combination of its $i$-graded parts, for $i$ ranging from 0 to 4 ; the $i$-graded part of $\mathbf{A}$ is denoted by $\langle\mathbf{A}\rangle_{i}$.

Lemma 3.1. 12] For any vectors $\mathbf{x}_{i} \in \mathbb{R}^{4}$,

$$
\begin{aligned}
2\left\langle\mathbf{x}_{1} \mathbf{x}_{2} \cdots \mathbf{x}_{2 k+1}\right\rangle_{1}= & \mathbf{x}_{1} \mathbf{x}_{2} \cdots \mathbf{x}_{2 k+1}+\mathbf{x}_{2 k+1} \cdots \mathbf{x}_{2} \mathbf{x}_{1} \\
2\left\langle\mathbf{x}_{1} \mathbf{x}_{2} \cdots \mathbf{x}_{2 k+1}\right\rangle_{3}= & \mathbf{x}_{1} \mathbf{x}_{2} \cdots \mathbf{x}_{2 k+1}-\mathbf{x}_{2 k+1} \cdots \mathbf{x}_{2} \mathbf{x}_{1}, \\
2\left\langle\mathbf{x}_{1} \mathbf{x}_{2} \cdots \mathbf{x}_{2 k}\right\rangle_{2}= & \mathbf{x}_{1} \mathbf{x}_{2} \cdots \mathbf{x}_{2 k}-\mathbf{x}_{2 k} \cdots \mathbf{x}_{2} \mathbf{x}_{1}, \\
4\left\langle\mathbf{x}_{1} \mathbf{x}_{2} \cdots \mathbf{x}_{2 k}\right\rangle_{4}= & \mathbf{x}_{1} \mathbf{x}_{2} \cdots \mathbf{x}_{2 k}+\mathbf{x}_{2 k} \cdots \mathbf{x}_{2} \mathbf{x}_{1} \\
& -\mathbf{x}_{2 k} \mathbf{x}_{1} \mathbf{x}_{2} \cdots \mathbf{x}_{2 k-1}-\mathbf{x}_{2 k-1} \cdots \mathbf{x}_{2} \mathbf{x}_{1} \mathbf{x}_{2 k}, \\
4\left\langle\mathbf{x}_{1} \mathbf{x}_{2} \cdots \mathbf{x}_{2 k}\right\rangle_{0}= & \mathbf{x}_{1} \mathbf{x}_{2} \cdots \mathbf{x}_{2 k}+\mathbf{x}_{2 k} \cdots \mathbf{x}_{2} \mathbf{x}_{1} \\
& +\mathbf{x}_{2 k} \mathbf{x}_{1} \mathbf{x}_{2} \cdots \mathbf{x}_{2 k-1}+\mathbf{x}_{2 k-1} \cdots \mathbf{x}_{2} \mathbf{x}_{1} \mathbf{x}_{2 k} .
\end{aligned}
$$

The reverse "†" in $C L\left(\mathbb{R}^{4}\right)$ is a linear operator defined as follows: for any $\mathbf{x}_{1} \mathbf{x}_{2} \cdots \mathbf{x}_{r}$ where $\mathbf{x}_{i} \in \mathbb{R}^{4},\left(\mathbf{x}_{1} \mathbf{x}_{2} \cdots \mathbf{x}_{r}\right)^{\dagger}:=\mathbf{x}_{r} \cdots \mathbf{x}_{2} \mathbf{x}_{1}$. The inverse of an element $\mathbf{A} \in C L\left(\mathbb{R}^{4}\right)$, denoted by $\mathbf{A}^{-1}$, is an element of $C L\left(\mathbb{R}^{4}\right)$ satisfying $\mathbf{A} \mathbf{A}^{-1}=1$. Not every element of $C L\left(\mathbb{R}^{4}\right)$ has inverse. For example, a vector of $\mathbb{R}^{4}$ is invertible if and only if it is nonzero.

For fixed orthonormal basis $\mathbf{e}_{0}, \mathbf{e}_{1}, \mathbf{e}_{2}, \mathbf{e}_{3}$ of $\mathbb{R}^{4}$, denote $\mathbf{e}_{p q r}:=\mathbf{e}_{p} \mathbf{e}_{q} \mathbf{e}_{r}$. Obviously $\mathbf{e}_{p q r}=\mathbf{e}_{p} \wedge \mathbf{e}_{q} \wedge \mathbf{e}_{r}$. The induced basis of $C L\left(\mathbb{R}^{4}\right)$ is

$$
1, \mathbf{e}_{0}, \mathbf{e}_{1}, \mathbf{e}_{2}, \mathbf{e}_{3}, \mathbf{e}_{01}, \mathbf{e}_{02}, \mathbf{e}_{03}, \mathbf{e}_{23}, \mathbf{e}_{13}, \mathbf{e}_{12}, \mathbf{e}_{012}, \mathbf{e}_{013}, \mathbf{e}_{023}, \mathbf{e}_{123}, \mathbf{e}_{0123} .
$$


We usually denote $\mathbf{I}_{4}:=\mathbf{e}_{0123}$. It is easy to see that $\mathbf{I}_{4}^{\dagger}=\mathbf{I}_{4}^{-1}=\mathbf{I}_{4}$, and for any $\mathbf{x} \in \mathbb{R}^{4}$,

$$
\mathbf{x} \mathbf{I}_{4}=-\mathbf{I}_{4} \mathbf{x} .
$$

In $C L\left(\mathbb{R}^{4}\right)$, the conjugate is a linear operator defined as follows: for any $\mathbf{A} \in C L\left(\mathbb{R}^{4}\right)$, its conjugate is

$$
\overline{\mathbf{A}}:=\mathbf{e}_{0} \mathbf{A e}_{0}
$$

For example, $\overline{\mathbf{I}_{4}}=-\mathbf{I}_{4}$, and for any $\mathbf{x}=\sum_{i=0}^{3} x_{i} \mathbf{e}_{i} \in \mathbb{R}^{4}, \overline{\mathbf{x}}=x_{0} \mathbf{e}_{0}-$ $\sum_{i=1}^{3} x_{i} \mathbf{e}_{i}$. Obviously $\overline{\mathbf{A B}}=\overline{\mathbf{A}} \overline{\mathbf{B}}$ for any $\mathbf{A}, \mathbf{B} \in C L\left(\mathbb{R}^{4}\right)$, and $\overline{\mathbf{a}}=\overline{\mathbf{a}}$ for any $\mathbf{a} \in \mathbb{H}$.

The dual of an element $\mathbf{A} \in C L\left(\mathbb{R}^{4}\right)$, denoted by $\mathbf{A}^{\sim}$, is defined by $\mathbf{A}^{\sim}:=\mathbf{A I}_{4}$. It is independent of the choice of the orthonormal basis $\mathbf{e}_{0}, \mathbf{e}_{1}, \mathbf{e}_{2}$, $\mathbf{e}_{3}$ of $\mathbb{R}^{4}$. The dual of $\mathbf{x}_{1} \wedge \mathbf{x}_{2} \wedge \mathbf{x}_{3} \wedge \mathbf{x}_{4}$ for $\mathbf{x}_{i} \in \mathbb{R}^{4}$ is usually denoted by $\left[\mathbf{x}_{1} \mathbf{x}_{2} \mathbf{x}_{3} \mathbf{x}_{4}\right]$, called the bracket of the four vectors.

For example, given $\mathbf{a}_{l}=u_{l}+x_{l} \mathbf{i}+y_{l} \mathbf{j}+z_{l} \mathbf{k} \in \mathbb{H}$ for $l=1 . .4$, then

$$
\left[\mathbf{a}_{1} \mathbf{a}_{2} \mathbf{a}_{3} \mathbf{a}_{4}\right]=\left|\begin{array}{llll}
u_{1} & x_{1} & y_{1} & z_{1} \\
u_{2} & x_{2} & y_{2} & z_{2} \\
u_{3} & x_{3} & y_{3} & z_{3} \\
u_{4} & x_{4} & y_{4} & z_{4}
\end{array}\right| .
$$

By Lemma 3.1, the bracket has a 4-termed representation in Clifford algebraic polynomial form.

Now we establish the shortest quaternionic polynomial forms of some typical Clifford algebraic expressions in $C L\left(\mathbb{R}^{4}\right)$, whose vector variables are images of quaternionic variables under $\mathbf{f}$.

Proposition 3.2. For any $\mathbf{a}_{1}, \mathbf{a}_{2} \in \mathbb{H}$,

$$
\dot{\mathbf{a}}_{1} \cdot \dot{\mathbf{a}}_{2}=\overline{\mathbf{a}}_{1} \cdot \dot{\mathbf{a}}_{2}=\frac{\mathbf{a}_{1} \overline{\mathbf{a}_{2}}+\mathbf{a}_{2} \overline{\mathbf{a}_{1}}}{2} \text {. }
$$

Proof. For any $\mathbf{a}_{l}=u_{l}+x_{l} \mathbf{i}+y_{l} \mathbf{j}+z_{l} \mathbf{k} \in \mathbb{H}$ where $l=1,2, \mathbf{a}_{1} \cdot \dot{\mathbf{a}}_{2}=\overline{\mathbf{a}}_{1} \cdot \overline{\mathbf{a}}_{2}=$ $u_{1} u_{2}+x_{1} x_{2}+y_{1} y_{2}+z_{1} z_{2}$ is the inner product in $\mathbb{R}^{4}$. The conclusion follows this and $\operatorname{Re}\left(\mathbf{a}_{1} \overline{\mathbf{a}_{2}}\right)=\operatorname{Re}\left(\mathbf{a}_{1}\right) \operatorname{Re}\left(\mathbf{a}_{2}\right)+\mathbf{f}\left(\operatorname{Im}\left(\mathbf{a}_{1}\right)\right) \cdot \mathbf{f}\left(\operatorname{Im}\left(\mathbf{a}_{2}\right)\right)$.

Lemma 3.3. For any $\mathbf{a}_{l} \in \mathbb{H}$ where $l=1 . .4$,

$$
\left[\dot{\mathbf{a}}_{1} \dot{\mathbf{a}}_{2} \dot{\mathbf{a}}_{3} \dot{\mathbf{a}}_{4}\right]=-\left[\overline{\mathbf{a}}_{1} \overline{\mathbf{a}}_{2} \overline{\mathbf{a}}_{3} \overline{\mathbf{a}}_{4}\right] \text {. }
$$

Proof. The conjugate operator defines an orthogonal transformation of determinant -1 in $\mathbb{R}^{4}$, so

$-\dot{\mathbf{a}}_{1} \wedge \mathbf{a}_{2} \wedge \mathbf{a}_{3} \wedge \dot{\mathbf{a}}_{4}=\overline{\mathbf{a}_{1} \wedge \dot{\mathbf{a}}_{2} \wedge \dot{\mathbf{a}}_{3} \wedge \dot{\mathbf{a}}_{4}}=\overline{\mathbf{a}_{1}} \wedge \overline{\mathbf{a}_{2}} \wedge \overline{\mathbf{a}_{3}} \wedge \overline{\mathbf{a}_{4}}=\overline{\mathbf{a}}_{1} \wedge \overline{\mathbf{a}}_{2} \wedge \overline{\mathbf{a}}_{3} \wedge \overline{\mathbf{a}}_{4}$.

For any $\mathbf{a}_{l} \in \mathbb{H}$, define the following elements in $\mathbb{H}$ :

$$
\begin{aligned}
& \mathbf{a}_{p q r}^{\sim}:=\mathbf{f}^{-1}\left(\left(\dot{\mathbf{a}}_{p} \wedge \mathbf{a}_{q} \wedge \mathbf{a}_{r}\right)^{\sim}\right), \\
& \overline{\mathbf{a}}_{p q r}^{\sim}:=\mathbf{f}^{-1}\left(\left(\overline{\mathbf{a}}_{p} \wedge \overline{\mathbf{a}}_{q} \wedge \overline{\mathbf{a}}_{r}\right)^{\sim}\right) .
\end{aligned}
$$


By Lemma 3.1. $\mathbf{a}_{p} \wedge \dot{\mathbf{a}}_{q} \wedge \mathbf{a}_{r}$ has 2-termed representation in the form of a Clifford algebraic polynomial. The same number of terms is expected for each of (3.8) in the form of a quaternionic polynomial. To obtain such a form we need to not only embed $\mathbb{H}$ into $C L\left(\mathbb{R}^{4}\right)$, but also project $C L\left(\mathbb{R}^{4}\right)$ onto $\mathbb{H}$; they are the two maps $\iota$ and $\pi$ below.

Definition 3.4. Denote by $H$ the 4-dimensional linear subspace of $C L\left(\mathbb{R}^{4}\right)$ spanned by $1, \mathbf{e}_{23}, \mathbf{e}_{13}, \mathbf{e}_{12}$. Define a linear isomorphism $\iota: H \mapsto \mathbb{H}$ :

$$
\iota(1)=1, \quad \iota\left(\mathbf{e}_{23}\right)=-\mathbf{i}, \quad \iota\left(\mathbf{e}_{13}\right)=\mathbf{j}, \quad \iota\left(\mathbf{e}_{12}\right)=-\mathbf{k} .
$$

Let $\pi_{H}$ be the restriction of $C L\left(\mathbb{R}^{4}\right)$ to subspace $H$. Define

$$
\begin{aligned}
\pi: C L\left(\mathbb{R}^{4}\right) & \rightarrow \mathbb{H} \\
\mathbf{A} & \mapsto\left(\iota \circ \pi_{H}\right)\left(\mathbf{A}\left(1+\mathbf{e}_{0}\right)\left(1-\mathbf{I}_{4}\right)\right)
\end{aligned}
$$

While $H=\left\langle 1, \mathbf{e}_{23}, \mathbf{e}_{13}, \mathbf{e}_{12}\right\rangle_{\mathbb{R}}$, we have

$$
\begin{aligned}
& \mathbf{e}_{0} H=\left\langle\mathbf{e}_{0}, \mathbf{e}_{023}, \mathbf{e}_{013}, \mathbf{e}_{012}\right\rangle_{\mathbb{R}}, \\
& \mathbf{I}_{4} H=\left\langle\mathbf{e}_{0123}, \mathbf{e}_{01}, \mathbf{e}_{02}, \mathbf{e}_{03}\right\rangle_{\mathbb{R}}, \\
& \mathbf{e}_{0} \mathbf{I}_{4} H=\left\langle\mathbf{e}_{123}, \mathbf{e}_{1}, \mathbf{e}_{2}, \mathbf{e}_{3}\right\rangle_{\mathbb{R}} .
\end{aligned}
$$

Then $C L\left(\mathbb{R}^{4}\right)=H \oplus \mathbf{e}_{0} H \oplus \mathbf{I}_{4} H \oplus \mathbf{e}_{0} \mathbf{I}_{4} H$. By definition,

$$
\begin{aligned}
& \left\{1, \quad \mathbf{e}_{0}, \quad \mathbf{e}_{123}, \quad-\mathbf{e}_{0123}\right\} \stackrel{\pi}{\rightarrow} 1 ; \\
& \left\{\mathbf{e}_{1},-\mathbf{e}_{01},-\mathbf{e}_{23}, \quad-\mathbf{e}_{023}\right\} \quad \stackrel{\pi}{\rightarrow} \mathbf{i} ; \\
& \left\{\mathbf{e}_{2},-\mathbf{e}_{02}, \quad \mathbf{e}_{13}, \quad \mathbf{e}_{013}\right\} \quad \stackrel{\pi}{\rightarrow} \mathbf{j} ; \\
& \left\{\mathbf{e}_{3},-\mathbf{e}_{03},-\mathbf{e}_{12},-\mathbf{e}_{012}\right\} \stackrel{\pi}{\rightarrow} \mathbf{k} .
\end{aligned}
$$

For any $\mathbf{a} \in \mathbb{H}$, obviously $\pi(\mathbf{a})=\mathbf{a}$, so $\pi \circ \mathbf{f}=\mathrm{id}$ in $\mathbb{H}$. Furthermore, $\pi$ is a linear isomorphism from any of $H, \mathbf{e}_{0} H, \mathbf{I}_{4} H, \mathbf{e}_{0} \mathbf{I}_{4} H$ to $\mathbb{H}$.

Let $K_{-}$be the odd elements of $\operatorname{ker}(\pi) \subset C L\left(\mathbb{R}^{4}\right)$. By (3.12), $K_{-}$is a 4-dimensional real space spanned by

$$
\begin{aligned}
& \mathbf{e}_{0}-\mathbf{e}_{123}=\mathbf{e}_{0}\left(1-\mathbf{I}_{4}\right), \\
& \mathbf{e}_{1}+\mathbf{e}_{023}=\mathbf{e}_{1}\left(1-\mathbf{I}_{4}\right), \\
& \mathbf{e}_{2}-\mathbf{e}_{013}=\mathbf{e}_{2}\left(1-\mathbf{I}_{4}\right), \\
& \mathbf{e}_{3}+\mathbf{e}_{012}=\mathbf{e}_{3}\left(1-\mathbf{I}_{4}\right) .
\end{aligned}
$$

So

$$
K_{-}=\mathbb{R}^{4}\left(1-\mathbf{I}_{4}\right)=\left\langle\mathbf{e}_{l}\left(1-\mathbf{I}_{4}\right), l=0 . .3\right\rangle_{\mathbb{R}} .
$$

Proposition 3.5. Let $\mathbf{A}, \mathbf{B} \in C L\left(\mathbb{R}^{4}\right)$, then

$$
\pi(\mathbf{A B})= \begin{cases}\pi(\mathbf{A}) \pi(\mathbf{B}), & \text { if } \mathbf{A} \text { is even } \\ \pi(\mathbf{A}) \pi(\overline{\mathbf{B}}), & \text { if } \mathbf{A} \text { is odd }\end{cases}
$$

In particular, $\pi(\mathbf{a} \mathbf{b})=\mathbf{a} \overline{\mathbf{b}}$ for any $\mathbf{a}, \mathbf{b} \in \mathbb{H}$.

Proof. (1). When $\mathbf{A}$ is even, let $\mathbf{A}=A_{0}+\mathbf{A}_{v}+D_{0} \mathbf{I}_{4}+\mathbf{D}_{v} \mathbf{I}_{4}$, where $A_{0}, D_{0}$ are scalars, and $\mathbf{A}_{v}, \mathbf{D}_{v} \in\left\langle\mathbf{e}_{23}, \mathbf{e}_{13}, \mathbf{e}_{12}\right\rangle_{\mathbb{R}}$. Then $\pi(\mathbf{A})=\iota\left(A_{0}+\mathbf{A}_{v}-D_{0}-\mathbf{D}_{v}\right)$. 
If $\mathbf{B}$ is even, let $\mathbf{B}=B_{0}+\mathbf{B}_{v}+C_{0} \mathbf{I}_{4}+\mathbf{C}_{v} \mathbf{I}_{4}$, where $B_{0}, C_{0}$ are scalars, and $\mathbf{B}_{v}, \mathbf{C}_{v} \in\left\langle\mathbf{e}_{23}, \mathbf{e}_{13}, \mathbf{e}_{12}\right\rangle_{\mathbb{R}}$. Then $\pi(\mathbf{B})=\iota\left(B_{0}+\mathbf{B}_{v}-C_{0}-\mathbf{C}_{v}\right)$. By

$$
\begin{aligned}
\mathbf{A B}= & A_{0} B_{0}+A_{0} \mathbf{B}_{v}+A_{0} C_{0} \mathbf{I}_{4}+A_{0} \mathbf{C}_{v} \mathbf{I}_{4} \\
& +B_{0} \mathbf{A}_{v}+\mathbf{A}_{v} \mathbf{B}_{v}+C_{0} \mathbf{A}_{v} \mathbf{I}_{4}+\mathbf{A}_{v} \mathbf{C}_{v} \mathbf{I}_{4} \\
& +B_{0} D_{0} \mathbf{I}_{4}+D_{0} \mathbf{I}_{4} \mathbf{B}_{v}+C_{0} D_{0}+D_{0} \mathbf{C}_{v} \\
& +B_{0} \mathbf{D}_{v} \mathbf{I}_{4}+\mathbf{D}_{v} \mathbf{B}_{v} \mathbf{I}_{4}+C_{0} \mathbf{D}_{v}+\mathbf{D}_{v} \mathbf{C}_{v}
\end{aligned}
$$

we have

$$
\begin{aligned}
\pi(\mathbf{A B})= & \iota\left(A_{0} B_{0}+A_{0} \mathbf{B}_{v}-A_{0} C_{0}-A_{0} \mathbf{C}_{v}\right. \\
& +B_{0} \mathbf{A}_{v}+\mathbf{A}_{v} \mathbf{B}_{v}-C_{0} \mathbf{A}_{v}-\mathbf{A}_{v} \mathbf{C}_{v} \\
& +C_{0} D_{0}+D_{0} \mathbf{C}_{v}-B_{0} D_{0}-D_{0} \mathbf{B}_{v} \\
& \left.-B_{0} \mathbf{D}_{v}-\mathbf{D}_{v} \mathbf{B}_{v}+C_{0} \mathbf{D}_{v}+\mathbf{D}_{v} \mathbf{C}_{v}\right) \\
= & \pi(\mathbf{A}) \iota\left(B_{0}+\mathbf{B}_{v}-C_{0}-\mathbf{C}_{v}\right) \\
= & \pi(\mathbf{A}) \pi(\mathbf{B})
\end{aligned}
$$

If $\mathbf{B}$ is odd, let $\mathbf{B}=b_{0} \mathbf{e}_{0}+\mathbf{b}_{v}+c_{0} \mathbf{e}_{0} \mathbf{I}_{4}+\mathbf{c}_{v} \mathbf{I}_{4}$, where $b_{0}, c_{0}$ are scalars, and $\mathbf{b}_{v}, \mathbf{c}_{v} \in\left\langle\mathbf{e}_{1}, \mathbf{e}_{2}, \mathbf{e}_{3}\right\rangle_{\mathbb{R}}$. Then $\pi(\mathbf{B})=\iota\left(b_{0}-\mathbf{b}_{v} \mathbf{e}_{0} \mathbf{I}_{4}+c_{0}-\mathbf{c}_{v} \mathbf{e}_{0} \mathbf{I}_{4}\right)$. By

$$
\begin{aligned}
\mathbf{A B}= & A_{0} b_{0} \mathbf{e}_{0}+A_{0} \mathbf{b}_{v}+A_{0} c_{0} \mathbf{e}_{0} \mathbf{I}_{4}+A_{0} \mathbf{c}_{v} \mathbf{I}_{4} \\
& +b_{0} \mathbf{A}_{v} \mathbf{e}_{0}+\mathbf{A}_{v} \mathbf{b}_{v}+c_{0} \mathbf{A}_{v} \mathbf{e}_{0} \mathbf{I}_{4}+\mathbf{A}_{v} \mathbf{c}_{v} \mathbf{I}_{4} \\
& -b_{0} D_{0} \mathbf{e}_{0} \mathbf{I}_{4}+D_{0} \mathbf{I}_{4} \mathbf{b}_{v}-c_{0} D_{0} \mathbf{e}_{0}-D_{0} \mathbf{c}_{v} \\
& -b_{0} \mathbf{D}_{v} \mathbf{e}_{0} \mathbf{I}_{4}+\mathbf{D}_{v} \mathbf{I}_{4} \mathbf{b}_{v}-c_{0} \mathbf{D}_{v} \mathbf{e}_{0}-\mathbf{D}_{v} \mathbf{c}_{v}
\end{aligned}
$$

we have

$$
\begin{aligned}
\pi(\mathbf{A B})= & \iota\left(A_{0} b_{0}-A_{0} \mathbf{c}_{v} \mathbf{e}_{0} \mathbf{I}_{4}-A_{0} \mathbf{b}_{v} \mathbf{e}_{0} \mathbf{I}_{4}+A_{0} c_{0}\right. \\
& +b_{0} \mathbf{A}_{v}-\mathbf{A}_{v} \mathbf{c}_{v} \mathbf{e}_{0} \mathbf{I}_{4}-\mathbf{A}_{v} \mathbf{b}_{v} \mathbf{e}_{0} \mathbf{I}_{4}+c_{0} \mathbf{A}_{v} \\
& +D_{0} \mathbf{b}_{v} \mathbf{e}_{0} \mathbf{I}_{4}-c_{0} D_{0}-b_{0} D_{0}+D_{0} \mathbf{c}_{v} \mathbf{e}_{0} \mathbf{I}_{4} \\
& \left.+\mathbf{D}_{v} \mathbf{b}_{v} \mathbf{e}_{0} \mathbf{I}_{4}-c_{0} \mathbf{D}_{v}-b_{0} \mathbf{D}_{v}+\mathbf{D}_{v} \mathbf{c}_{v} \mathbf{e}_{0} \mathbf{I}_{4}\right) \\
= & \pi(\mathbf{A}) \iota\left(b_{0}-\mathbf{b}_{v} \mathbf{e}_{0} \mathbf{I}_{4}+c_{0}-\mathbf{c}_{v} \mathbf{e}_{0} \mathbf{I}_{4}\right) \\
= & \pi(\mathbf{A}) \pi(\mathbf{B}) .
\end{aligned}
$$

(2). When $\mathbf{A}$ is odd, let $\mathbf{A}=a_{0} \mathbf{e}_{0}+\mathbf{a}_{v}+d_{0} \mathbf{e}_{0} \mathbf{I}_{4}+\mathbf{d}_{v} \mathbf{I}_{4}$, where $a_{0}, d_{0}$ are scalars, and $\mathbf{a}_{v}, \mathbf{d}_{v} \in\left\langle\mathbf{e}_{1}, \mathbf{e}_{2}, \mathbf{e}_{3}\right\rangle_{\mathbb{R}}$. Then $\pi(\mathbf{A})=\iota\left(a_{0}-\mathbf{a}_{v} \mathbf{e}_{0} \mathbf{I}_{4}+d_{0}-\mathbf{d}_{v} \mathbf{e}_{0} \mathbf{I}_{4}\right)$.

If $\mathbf{B}$ is even, let $\mathbf{B}=B_{0}+\mathbf{B}_{v}+C_{0} \mathbf{I}_{4}+\mathbf{C}_{v} \mathbf{I}_{4}$, where $B_{0}, C_{0}$ are scalars, and $\mathbf{B}_{v}, \mathbf{C}_{v} \in\left\langle\mathbf{e}_{23}, \mathbf{e}_{13}, \mathbf{e}_{12}\right\rangle_{\mathbb{R}}$. Then $\overline{\mathbf{B}}=B_{0}+\mathbf{B}_{v}-C_{0} \mathbf{I}_{4}-\mathbf{C}_{v} \mathbf{I}_{4}$, and $\pi(\overline{\mathbf{B}})=$ $\iota\left(B_{0}+\mathbf{B}_{v}+C_{0}+\mathbf{C}_{v}\right)$. By

$$
\begin{aligned}
\mathbf{A B}= & a_{0} B_{0} \mathbf{e}_{0}+a_{0} \mathbf{e}_{0} \mathbf{B}_{v}+a_{0} C_{0} \mathbf{e}_{0} \mathbf{I}_{4}+a_{0} \mathbf{C}_{v} \mathbf{e}_{0} \mathbf{I}_{4} \\
& +B_{0} \mathbf{a}_{v}+\mathbf{a}_{v} \mathbf{B}_{v}+C_{0} \mathbf{a}_{v} \mathbf{I}_{4}+\mathbf{a}_{v} \mathbf{C}_{v} \mathbf{I}_{4} \\
& +d_{0} B_{0} \mathbf{e}_{0} \mathbf{I}_{4}+d_{0} \mathbf{e}_{0} \mathbf{I}_{4} \mathbf{B}_{v}+d_{0} C_{0} \mathbf{e}_{0}+d_{0} \mathbf{C}_{v} \mathbf{e}_{0} \\
& +B_{0} \mathbf{d}_{v} \mathbf{I}_{4}+\mathbf{d}_{v} \mathbf{I}_{4} \mathbf{B}_{v}+C_{0} \mathbf{d}_{v}+\mathbf{d}_{v} \mathbf{C}_{v}
\end{aligned}
$$


we have

$$
\begin{aligned}
\pi(\mathbf{A B})= & \iota\left(a_{0} B_{0}+a_{0} \mathbf{B}_{v}+a_{0} C_{0}+a_{0} \mathbf{C}_{v}\right. \\
& -B_{0} \mathbf{a}_{v} \mathbf{e}_{0} \mathbf{I}_{4}-\mathbf{a}_{v} \mathbf{B}_{v} \mathbf{e}_{0} \mathbf{I}_{4}-C_{0} \mathbf{a}_{v} \mathbf{e}_{0} \mathbf{I}_{4}-\mathbf{a}_{v} \mathbf{C}_{v} \mathbf{e}_{0} \mathbf{I}_{4} \\
& +d_{0} B_{0}+d_{0} \mathbf{B}_{v}+d_{0} C_{0}+d_{0} \mathbf{C}_{v} \\
& \left.-B_{0} \mathbf{d}_{v} \mathbf{e}_{0} \mathbf{I}_{4}-\mathbf{d}_{v} \mathbf{B}_{v} \mathbf{e}_{0} \mathbf{I}_{4}-C_{0} \mathbf{d}_{v} \mathbf{e}_{0} \mathbf{I}_{4}-\mathbf{d}_{v} \mathbf{C}_{v} \mathbf{e}_{0} \mathbf{I}_{4}\right) \\
= & \pi(\mathbf{A}) \iota\left(B_{0}+\mathbf{B}_{v}+C_{0}+\mathbf{C}_{v}\right) \\
= & \pi(\mathbf{A}) \pi(\overline{\mathbf{B}}) .
\end{aligned}
$$

If $\mathbf{B}$ is odd, let $\mathbf{B}=b_{0} \mathbf{e}_{0}+\mathbf{b}_{v}+\mathbf{c}_{v} \mathbf{I}_{4}+c_{0} \mathbf{e}_{0} \mathbf{I}_{4}$, where $b_{0}, c_{0}$ are scalars and $\mathbf{b}_{v}, \mathbf{c}_{v} \in\left\langle\mathbf{e}_{1}, \mathbf{e}_{2}, \mathbf{e}_{3}\right\rangle_{\mathbb{R}}$. Then $\overline{\mathbf{B}}=b_{0} \mathbf{e}_{0}-\mathbf{b}_{v}+\mathbf{c}_{v} \mathbf{I}_{4}-c_{0} \mathbf{e}_{0} \mathbf{I}_{4}$, and $\pi(\overline{\mathbf{B}})=$ $\iota\left(b_{0}+\mathbf{b}_{v} \mathbf{e}_{0} \mathbf{I}_{4}-c_{0}-\mathbf{c}_{v} \mathbf{e}_{0} \mathbf{I}_{4}\right)$. By

$$
\begin{aligned}
\mathbf{A B}= & a_{0} b_{0}+a_{0} \mathbf{e}_{0} \mathbf{b}_{v}+a_{0} c_{0} \mathbf{I}_{4}-a_{0} \mathbf{c}_{v} \mathbf{e}_{0} \mathbf{I}_{4} \\
& +b_{0} \mathbf{a}_{v} \mathbf{e}_{0}+\mathbf{a}_{v} \mathbf{b}_{v}+c_{0} \mathbf{a}_{v} \mathbf{e}_{0} \mathbf{I}_{4}+\mathbf{a}_{v} \mathbf{c}_{v} \mathbf{I}_{4} \\
& -d_{0} b_{0} \mathbf{I}_{4}+d_{0} \mathbf{e}_{0} \mathbf{I}_{4} \mathbf{b}_{v}-d_{0} c_{0}+d_{0} \mathbf{c}_{v} \mathbf{e}_{0} \\
& -b_{0} \mathbf{d}_{v} \mathbf{e}_{0} \mathbf{I}_{4}+\mathbf{d}_{v} \mathbf{I}_{4} \mathbf{b}_{v}-c_{0} \mathbf{d}_{v} \mathbf{e}_{0}-\mathbf{d}_{v} \mathbf{c}_{v}
\end{aligned}
$$

we have

$$
\begin{aligned}
\pi(\mathbf{A B})= & a_{0} b_{0}-a_{0} \mathbf{c}_{v} \mathbf{e}_{0} \mathbf{I}_{4}+a_{0} \mathbf{b}_{v} \mathbf{e}_{0} \mathbf{I}_{4}-a_{0} c_{0} \\
& -b_{0} \mathbf{a}_{v} \mathbf{e}_{0} \mathbf{I}_{4}+\mathbf{a}_{v} \mathbf{b}_{v}+c_{0} \mathbf{a}_{v} \mathbf{e}_{0} \mathbf{I}_{4}-\mathbf{a}_{v} \mathbf{c}_{v} \\
& +d_{0} b_{0}-d_{0} \mathbf{c}_{v} \mathbf{e}_{0} \mathbf{I}_{4}+d_{0} \mathbf{e}_{0} \mathbf{I}_{4} \mathbf{b}_{v}-d_{0} c_{0} \\
& -b_{0} \mathbf{d}_{v} \mathbf{e}_{0} \mathbf{I}_{4}-\mathbf{d}_{v} \mathbf{c}_{v}+\mathbf{d}_{v} \mathbf{b}_{v}+c_{0} \mathbf{d}_{v} \mathbf{e}_{0} \mathbf{I}_{4} \\
= & \pi(\mathbf{A}) \iota\left(b_{0}+\mathbf{b}_{v} \mathbf{e}_{0} \mathbf{I}_{4}-c_{0}-\mathbf{c}_{v} \mathbf{e}_{0} \mathbf{I}_{4}\right) \\
= & \pi(\mathbf{A}) \pi(\overline{\mathbf{B}})
\end{aligned}
$$

Corollary 3.6. For any $r>0$ and $\mathbf{a}_{l} \in \mathbb{H}$,

$$
\pi\left(\dot{\mathbf{a}}_{1} \dot{\mathbf{a}}_{2} \cdots \dot{\mathbf{a}}_{r}\right)= \begin{cases}\mathbf{a}_{1} \overline{\mathbf{a}}_{2} \mathbf{a}_{3} \overline{\mathbf{a}}_{4} \cdots \mathbf{a}_{r-1} \overline{\mathbf{a}}_{r}, & \text { if } r \text { is even } \\ \mathbf{a}_{1} \overline{\mathbf{a}}_{2} \mathbf{a}_{3} \overline{\mathbf{a}}_{4} \cdots \overline{\mathbf{a}}_{r-1} \mathbf{a}_{r}, & \text { if } r \text { is odd }\end{cases}
$$

Proposition 3.7. For any $\mathbf{a}_{l} \in \mathbb{H}$,

$$
\left[\dot{\mathbf{a}}_{1} \dot{\mathbf{a}}_{2} \dot{\mathbf{a}}_{3} \dot{\mathbf{a}}_{4}\right]=-\frac{1}{4}\left(\mathbf{a}_{1} \overline{\mathbf{a}}_{2} \mathbf{a}_{3} \overline{\mathbf{a}}_{4}+\mathbf{a}_{4} \overline{\mathbf{a}}_{3} \mathbf{a}_{2} \overline{\mathbf{a}}_{1}-\mathbf{a}_{4} \overline{\mathbf{a}}_{1} \mathbf{a}_{2} \overline{\mathbf{a}}_{3}-\mathbf{a}_{3} \overline{\mathbf{a}}_{2} \mathbf{a}_{1} \overline{\mathbf{a}}_{4}\right) .
$$

Proof. By definition, $\mathbf{a}_{1} \wedge \mathbf{a}_{2} \wedge \mathbf{a}_{3} \wedge \mathbf{a}_{4}=\left[\mathbf{a}_{1} \mathbf{a}_{2} \mathbf{a}_{3} \mathbf{a}_{4}\right] \mathbf{I}_{4}$. On the other hand, by

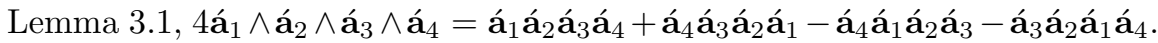
So

$$
\begin{aligned}
{\left[\dot{\mathbf{a}}_{1} \dot{\mathbf{a}}_{2} \dot{\mathbf{a}}_{3} \dot{\mathbf{a}}_{4}\right] } & =\pi\left(\dot{\mathbf{a}}_{1} \wedge \mathbf{a}_{2} \wedge \dot{\mathbf{a}}_{3} \wedge \mathbf{a}_{4}\right) \pi\left(\mathbf{I}_{4}\right) \\
& =-\frac{1}{4}\left(\mathbf{a}_{1} \overline{\mathbf{a}}_{2} \mathbf{a}_{3} \overline{\mathbf{a}}_{4}+\mathbf{a}_{4} \overline{\mathbf{a}}_{3} \mathbf{a}_{2} \overline{\mathbf{a}}_{1}-\mathbf{a}_{4} \overline{\mathbf{a}}_{1} \mathbf{a}_{2} \overline{\mathbf{a}}_{3}-\mathbf{a}_{3} \overline{\mathbf{a}}_{2} \mathbf{a}_{1} \overline{\mathbf{a}}_{4}\right) .
\end{aligned}
$$


Proposition 3.8. For any $\mathbf{a}_{1}, \mathbf{a}_{2}, \mathbf{a}_{3} \in \mathbb{H}$,

$$
\mathbf{a}_{123}^{\sim}=\frac{1}{2}\left(\mathbf{a}_{1} \overline{\mathbf{a}}_{2} \mathbf{a}_{3}-\mathbf{a}_{3} \overline{\mathbf{a}}_{2} \mathbf{a}_{1}\right) \text {. }
$$

Furthermore,

$$
\overline{\mathbf{a}}_{123}^{\sim}=-\overline{\mathbf{a}_{123}^{\sim}}
$$

Proof. By Lemma 3.1 $2\left(\dot{a}_{1} \wedge \dot{\mathbf{a}}_{2} \wedge \mathbf{a}_{3}\right)^{\sim}=\left(\dot{a}_{1} \dot{\mathbf{a}}_{2} \mathbf{a}_{3}-\mathbf{a}_{3} \dot{\mathbf{a}}_{2} \mathbf{a}_{1}\right) \mathbf{I}_{4}$. So

$$
2 \mathbf{a}_{123}^{\sim}=2 \pi\left(\dot{\mathbf{a}}_{1} \wedge \dot{\mathbf{a}}_{2} \wedge \dot{\mathbf{a}}_{3}\right) \pi\left(\overline{\mathbf{I}_{4}}\right)=\pi\left(\dot{\mathbf{a}}_{1} \mathbf{a}_{2} \dot{\mathbf{a}}_{3}-\dot{\mathbf{a}}_{3} \dot{\mathbf{a}}_{2} \dot{\mathbf{a}}_{1}\right)=\mathbf{a}_{1} \overline{\mathbf{a}}_{2} \mathbf{a}_{3}-\mathbf{a}_{3} \overline{\mathbf{a}}_{2} \mathbf{a}_{1} .
$$

Similarly, $2 \overline{\mathbf{a}}_{123}^{\sim}=\overline{\mathbf{a}}_{1} \mathbf{a}_{2} \overline{\mathbf{a}}_{3}-\overline{\mathbf{a}}_{3} \mathbf{a}_{2} \overline{\mathbf{a}}_{1}=-2 \overline{\mathbf{a}_{123}^{\sim}}$.

\section{The associated real linear system approach}

The revised starting form (1.11) can be written as a real linear system Aq́ = $\mathbf{d}$, where matrix $\mathbf{A}$ is given by (2.6). The solution is $\mathbf{q}=\operatorname{adj}(\mathbf{A}) \mathbf{d} / \operatorname{det}(\mathbf{A})$ under the non-degeneracy condition $\operatorname{det}(\mathbf{A}) \neq 0$.

\section{Theorem 4.1.}

$$
\operatorname{det}(\mathbf{A})=2 \sum_{i, j=0}^{3}\left(\dot{\mathbf{a}}_{i} \cdot \dot{\mathbf{a}}_{j}\right)^{2}-\left(\sum_{i=0}^{3} \mathbf{a}_{i} \cdot \dot{\mathbf{a}}_{i}\right)^{2}-8\left[\dot{\mathbf{a}}_{0} \mathbf{a}_{1} \dot{\mathbf{a}}_{2} \mathbf{a}_{3}\right] .
$$

Proof. After direct expansion and simplification by Maple, we get that $\operatorname{det}(\mathbf{A})$ is a polynomial of 196 terms in the indeterminates $a_{i j}$ for $i, j=0 . .3$. The terms can be categorized into two groups. The first group $G_{1}$ is the sum of terms of the form $a_{i j}^{4}$ or $a_{u v}^{2} a_{k l}^{2}$; the second group $G_{2}$ is the sum of the other terms. All terms of $G_{2}$ are square-free.

We first consider $G_{1}$. Denote

$$
\mathbf{N}_{1}=\left(\begin{array}{llll}
1 & 1 & 1 & 1 \\
1 & 1 & 1 & 1 \\
1 & 1 & 1 & 1 \\
1 & 1 & 1 & 1
\end{array}\right), \quad \mathbf{N}_{2}=\left(\begin{array}{rrrr}
1 & -1 & -1 & -1 \\
-1 & 1 & -1 & -1 \\
-1 & -1 & 1 & -1 \\
-1 & -1 & -1 & 1
\end{array}\right)
$$

Further denote

$$
\mathbf{v}_{l}^{T}:=\left(a_{l 0}^{2}, a_{l 1}^{2}, a_{l 2}^{2}, a_{l 3}^{2}\right), \text { for } l=0 . .3
$$

It is easy to prove that for any $i, j \in\{0,1,2,3\}$,

$$
\mathbf{v}_{i}^{T} \mathbf{N}_{1} \mathbf{v}_{j}=\mathbf{a}_{i}^{2} \mathbf{a}_{j}^{2}, \quad \mathbf{v}_{i}^{T} \mathbf{N}_{2} \mathbf{v}_{j}=2 \mathbf{v}_{i}^{T} \mathbf{v}_{j}-\mathbf{v}_{i}^{T} \mathbf{N}_{1} \mathbf{v}_{j}
$$


By direct verification, we confirm that $G_{1}$ equals

$$
\begin{aligned}
& \left(\begin{array}{llll}
\mathbf{v}_{0}^{T} & \mathbf{v}_{1}^{T} & \mathbf{v}_{2}^{T} & \mathbf{v}_{3}^{T}
\end{array}\right)\left(\begin{array}{llll}
\mathbf{N}_{1} & \mathbf{N}_{2} & \mathbf{N}_{2} & \mathbf{N}_{2} \\
\mathbf{N}_{2} & \mathbf{N}_{1} & \mathbf{N}_{2} & \mathbf{N}_{2} \\
\mathbf{N}_{2} & \mathbf{N}_{2} & \mathbf{N}_{1} & \mathbf{N}_{2} \\
\mathbf{N}_{2} & \mathbf{N}_{2} & \mathbf{N}_{2} & \mathbf{N}_{1}
\end{array}\right)\left(\begin{array}{l}
\mathbf{v}_{0} \\
\mathbf{v}_{1} \\
\mathbf{v}_{2} \\
\mathbf{v}_{3}
\end{array}\right) \\
= & \sum_{i=0}^{3} \mathbf{v}_{i}^{T} \mathbf{N}_{1} \mathbf{v}_{i}+2 \sum_{0 \leq j<k \leq 3} \mathbf{v}_{j}^{T} \mathbf{N}_{2} \mathbf{v}_{k} \\
= & \sum_{i=0}^{3} \mathbf{a}_{i}^{2}-2 \sum_{0 \leq j<k \leq 3} \mathbf{a}_{j}^{2} \mathbf{a}_{k}^{2}+4 \sum_{0 \leq j<k \leq 3} \mathbf{v}_{j}^{T} \mathbf{v}_{k} .
\end{aligned}
$$

Again by direct verification, we confirm that $G_{2}$ equals

$$
-8\left[\dot{\mathbf{a}}_{0} \mathbf{a}_{1} \mathbf{a}_{2} \mathbf{a}_{3}\right]+4 \sum_{0 \leq i<j \leq 3}\left(\dot{\mathbf{a}}_{i} \cdot \dot{\mathbf{a}}_{j}\right)^{2}-4 \sum_{0 \leq i<j \leq 3} \mathbf{v}_{i}^{T} \mathbf{v}_{j} .
$$

By (4.2) and (4.3), we get (4.1).

Next consider the expression of the adjugate $\operatorname{adj}(\mathbf{A})$ of $\mathbf{A}$. Denote

$$
\begin{aligned}
& \Lambda:=\sum_{l=0}^{3} \mathbf{a}_{l}^{2} \quad=\sum_{l=0}^{3} \mathbf{a}_{l} \overline{\mathbf{a}}_{l}, \\
& \operatorname{adj}\left(\mathbf{a}_{i}\right):=\sum_{l=0}^{3}\left(\dot{\mathbf{a}}_{i} \cdot \dot{\mathbf{a}}_{l}\right) \overline{\mathbf{a}}_{l}=\frac{1}{2} \sum_{l=0}^{3}\left(\mathbf{a}_{i} \overline{\mathbf{a}}_{l}+\mathbf{a}_{l} \overline{\mathbf{a}}_{i}\right) \overline{\mathbf{a}}_{l} .
\end{aligned}
$$

Theorem 4.2. With the notations introduced by (3.8) and (4.4),

$$
\begin{aligned}
\operatorname{adj}(\mathbf{A})= & \left(2 \overline{\mathbf{a}}_{123}^{\sim}+2 \operatorname{adj}\left(\overline{\mathbf{a}}_{0}\right)-\Lambda \overline{\mathbf{a}}_{0} \mid 1\right) \\
& -\left(-2 \overline{\mathbf{a}}_{023}^{\sim}+2 \operatorname{adj}\left(\overline{\mathbf{a}}_{1}\right)-\Lambda \overline{\mathbf{a}}_{1} \mid \mathbf{i}\right) \\
& -\left(2 \overline{\mathbf{a}}_{013}^{\sim}+2 \operatorname{adj}\left(\overline{\mathbf{a}}_{2}\right)-\Lambda \overline{\mathbf{a}}_{2} \mid \mathbf{j}\right) \\
& -\left(-2 \overline{\mathbf{a}}_{012}^{\sim}+2 \operatorname{adj}\left(\overline{\mathbf{a}}_{3}\right)-\Lambda \overline{\mathbf{a}}_{3} \mid \mathbf{k}\right) .
\end{aligned}
$$

Proof. The entries of $\operatorname{adj}(\mathbf{A})$ are algebraic minors of $\mathbf{A}$. Once we obtain them, then by Proposition 2.1,

$$
\operatorname{adj}(\mathbf{A})=\left(\mathbf{p}_{0} \mid 1\right)+\left(\mathbf{p}_{1} \mid \mathbf{i}\right)+\left(\mathbf{p}_{2} \mid \mathbf{j}\right)+\left(\mathbf{p}_{3} \mid \mathbf{k}\right),
$$

where the $\mathbf{p}_{l}$ satisfy (2.8) for $\left(m_{i j}\right)_{i, j=0 . .3}=\operatorname{adj}(\mathbf{A})$.

We first compute $\mathbf{p}_{0}=p_{00}+p_{01} \mathbf{i}+p_{02} \mathbf{j}+p_{03} \mathbf{k}$. By (2.8) and direct verification,

$$
\begin{aligned}
& p_{00}=a_{00}\left(\mathbf{a}_{0}^{2}-\mathbf{a}_{1}^{2}-\mathbf{a}_{2}^{2}-\mathbf{a}_{3}^{2}\right)+2 a_{10} \mathbf{a}_{0} \cdot \mathbf{a}_{1}+2 a_{20} \mathbf{a}_{0} \cdot \mathbf{a}_{2} \\
& +2 a_{30} \mathbf{a}_{0} \cdot \mathbf{a}_{3}-2\left[\mathbf{e}_{0} \mathbf{a}_{1} \mathbf{a}_{2} \mathbf{a}_{3}\right], \\
& p_{01}=-a_{01}\left(\dot{a}_{0}^{2}-\mathbf{a}_{1}^{2}-\mathbf{a}_{2}^{2}-\mathbf{a}_{3}^{2}\right)-2 a_{11} \dot{\mathbf{a}}_{0} \cdot \dot{\mathbf{a}}_{1}-2 a_{21} \mathbf{a}_{0} \cdot \mathbf{a}_{2} \\
& -2 a_{31} \dot{a}_{0} \cdot \mathbf{a}_{3}+2\left[\mathbf{e}_{1} \dot{a}_{1} \mathbf{a}_{2} \mathbf{a}_{3}\right], \\
& p_{02}=\left(\dot{a}_{0}^{2}-\mathbf{a}_{1}^{2}-\mathbf{a}_{2}^{2}-\mathbf{a}_{3}^{2}\right)-2 a_{12} \dot{a}_{0} \cdot \mathbf{a}_{1}-2 a_{22} \mathbf{a}_{0} \cdot \mathbf{a}_{2} \\
& -2 a_{32} \dot{a}_{0} \cdot \mathbf{a}_{3}+2\left[\mathbf{e}_{2} \mathbf{a}_{1} \mathbf{a}_{2} \mathbf{a}_{3}\right], \\
& p_{03}=-a_{03}\left(\dot{a}_{0}^{2}-\mathbf{a}_{1}^{2}-\dot{\mathbf{a}}_{2}^{2}-\mathbf{a}_{3}^{2}\right)-2 a_{13} \dot{a}_{0} \cdot \dot{\mathbf{a}}_{1}-2 a_{23} \dot{a}_{0} \cdot \mathbf{a}_{2} \\
& -2 a_{33} \dot{a}_{0} \cdot \dot{a}_{3}+2\left[\mathbf{e}_{3} \dot{a}_{1} \dot{a}_{2} \dot{a}_{3}\right] \text {. }
\end{aligned}
$$


So

$$
\begin{aligned}
\mathbf{p}_{0}= & \left(\dot{\mathbf{a}}_{0}^{2}-\dot{\mathbf{a}}_{1}^{2}-\dot{\mathbf{a}}_{2}^{2}-\dot{\mathbf{a}}_{3}^{2}\right) \overline{\mathbf{a}}_{0}+2\left(\dot{\mathbf{a}}_{0} \cdot \dot{\mathbf{a}}_{1}\right) \overline{\mathbf{a}}_{1}+2\left(\dot{\mathbf{a}}_{0} \cdot \dot{\mathbf{a}}_{2}\right) \dot{\mathbf{a}}_{2}+2\left(\dot{\mathbf{a}}_{0} \cdot \dot{\mathbf{a}}_{3}\right) \overline{\mathbf{a}}_{3} \\
& -2\left(\left[\mathbf{e}_{0} \mathbf{a}_{1} \mathbf{a}_{2} \mathbf{a}_{3}\right] \mathbf{e}_{0}-\left[\mathbf{e}_{1} \mathbf{a}_{1} \mathbf{a}_{2} \dot{\mathbf{a}}_{3}\right] \mathbf{e}_{1}-\left[\mathbf{e}_{2} \mathbf{a}_{1} \mathbf{a}_{2} \dot{\mathbf{a}}_{3}\right] \mathbf{e}_{2}-\left[\mathbf{e}_{3} \dot{\mathbf{a}}_{1} \dot{\mathbf{a}}_{2} \dot{\mathbf{a}}_{3}\right] \mathbf{e}_{3}\right) .
\end{aligned}
$$

Similarly, we get

$$
\begin{aligned}
& \mathbf{p}_{1}=\left(\mathbf{a}_{0}^{2}-\dot{\mathbf{a}}_{1}^{2}+\dot{\mathbf{a}}_{2}^{2}+\dot{\mathbf{a}}_{3}^{2}\right) \overline{\mathbf{a}}_{1}-2\left(\dot{\mathbf{a}}_{1} \cdot \dot{\mathbf{a}}_{0}\right) \overline{\mathbf{a}}_{0}-2\left(\dot{\mathbf{a}}_{1} \cdot \dot{\mathbf{a}}_{2}\right) \overline{\mathbf{a}}_{2}-2\left(\dot{\mathbf{a}}_{1} \cdot \dot{\mathbf{a}}_{3}\right) \overline{\mathbf{a}}_{3} \\
& -2\left(\left[\mathbf{e}_{0} \mathbf{a}_{0} \mathbf{a}_{2} \mathbf{a}_{3}\right] \mathbf{e}_{0}-\left[\mathbf{e}_{1} \mathbf{a}_{0} \mathbf{a}_{2} \mathbf{a}_{3}\right] \mathbf{e}_{1}-\left[\mathbf{e}_{2} \mathbf{a}_{0} \mathbf{a}_{2} \mathbf{a}_{3}\right] \mathbf{e}_{2}-\left[\mathbf{e}_{3} \mathbf{a}_{0} \mathbf{a}_{2} \dot{a}_{3}\right] \mathbf{e}_{3}\right), \\
& \mathbf{p}_{2}=\left(\mathbf{a}_{0}^{2}+\dot{\mathbf{a}}_{1}^{2}-\mathbf{a}_{2}^{2}+\dot{\mathbf{a}}_{3}^{2}\right) \overline{\mathbf{a}}_{2}+2\left(\dot{\mathbf{a}}_{2} \cdot \dot{\mathbf{a}}_{0}\right) \overline{\mathbf{a}}_{0}+2\left(\dot{\mathbf{a}}_{2} \cdot \dot{\mathbf{a}}_{1}\right) \overline{\mathbf{a}}_{1}+2\left(\dot{\mathbf{a}}_{2} \cdot \mathbf{a}_{3}\right) \overline{\mathbf{a}}_{3} \\
& +2\left(\left[\mathbf{e}_{0} \mathbf{a}_{0} \dot{\mathbf{a}}_{1} \mathbf{a}_{3}\right] \mathbf{e}_{0}-\left[\mathbf{e}_{1} \dot{\mathbf{a}}_{0} \mathbf{a}_{1} \dot{\mathbf{a}}_{3}\right] \mathbf{e}_{1}-\left[\mathbf{e}_{2} \dot{\mathbf{a}}_{0} \mathbf{a}_{1} \mathbf{a}_{3}\right] \mathbf{e}_{2}-\left[\mathbf{e}_{3} \dot{a}_{0} \dot{\mathbf{a}}_{1} \dot{\mathbf{a}}_{3}\right] \mathbf{e}_{3}\right), \\
& \mathbf{p}_{3}=\left(\dot{\mathbf{a}}_{0}^{2}-\mathbf{a}_{1}^{2}-\mathbf{a}_{2}^{2}-\mathbf{a}_{3}^{2}\right) \overline{\mathbf{a}}_{3}+2\left(\dot{\mathbf{a}}_{3} \cdot \dot{\mathbf{a}}_{0}\right) \overline{\mathbf{a}}_{0}+2\left(\dot{\mathbf{a}}_{3} \cdot \mathbf{a}_{2}\right) \overline{\mathbf{a}}_{1}+2\left(\mathbf{a}_{3} \cdot \mathbf{a}_{3}\right) \overline{\mathbf{a}}_{2} \\
& -2\left(\left[\mathbf{e}_{0} \dot{a}_{0} \dot{a}_{1} \dot{a}_{2}\right] \mathbf{e}_{0}-\left[\mathbf{e}_{1} \dot{\mathbf{a}}_{0} \dot{\mathbf{a}}_{1} \dot{\mathbf{a}}_{2}\right] \mathbf{e}_{1}-\left[\mathbf{e}_{2} \dot{\mathbf{a}}_{0} \dot{\mathbf{a}}_{1} \dot{\mathbf{a}}_{2}\right] \mathbf{e}_{2}-\left[\mathbf{e}_{3} \dot{a}_{0} \dot{a}_{1} \dot{a}_{2}\right] \mathbf{e}_{3}\right) \text {. }
\end{aligned}
$$

Substituting these expressions into (4.6), and using the notations introduced by (3.8) and (4.4), we get (4.5).

Now consider the general linear quaternionic equation (1.1). Let $\mathbf{b}_{p}=$ $b_{p 0}+b_{p 1} \mathbf{i}+b_{p 2} \mathbf{j}+b_{p 3} \mathbf{k}$ for $p=1 . . n-1$, and set

$$
\mathbf{a}_{i}:=\sum_{p=1}^{n-1} b_{p i} \mathbf{c}_{p}, \text { for } i \in\{0,1,2,3\}
$$

Then (1.1) is changed into the revised starting form (1.11).

Theorem 4.3. With the $\mathbf{a}_{i}$ taking values (4.9),

$$
\begin{aligned}
\operatorname{det}(\mathbf{A})= & 2 \sum_{p, q, r, s=1}^{n-1}\left(\dot{\mathbf{c}}_{p} \cdot \dot{\mathbf{c}}_{q}\right)\left(\dot{\mathbf{c}}_{r} \cdot \dot{\mathbf{c}}_{s}\right)\left(\dot{\mathbf{b}}_{p} \cdot \dot{\mathbf{b}}_{r}\right)\left(\dot{\mathbf{b}}_{q} \cdot \dot{\mathbf{b}}_{s}\right) \\
& -\left(\sum_{p, q=1}^{n-1}\left(\dot{\mathbf{c}}_{p} \cdot \dot{\mathbf{c}}_{q}\right)\left(\dot{\mathbf{b}}_{p} \cdot \dot{\mathbf{b}}_{q}\right)\right)^{2}-\frac{1}{3} \sum_{p, q, r, s=1}^{n-1}\left[\dot{\mathbf{c}}_{p} \dot{\mathbf{c}}_{q} \dot{\mathbf{c}}_{r} \dot{\mathbf{c}}_{s}\right]\left[\dot{\mathbf{b}}_{p} \dot{b}_{q} \dot{\mathbf{b}}_{r} \dot{\mathbf{b}}_{s}\right] .
\end{aligned}
$$

The solution of (1.1) is unique if and only if $\operatorname{det}(\mathbf{A}) \neq 0$, and the solution when $\operatorname{det}(\mathbf{A}) \neq 0$ is

$$
\mathbf{q}=\frac{-1}{3 \operatorname{det}(\mathbf{A})} \sum_{p, q, r=1}^{n-1}\left\{\overline{\mathbf{c}}_{p q r}^{\sim} \mathbf{d} \overline{\mathbf{b}}_{p q r}^{\sim}+3\left(\dot{\mathbf{c}}_{p} \cdot \dot{\mathbf{c}}_{q}\right)\left(\left(\dot{\mathbf{b}}_{p} \cdot \dot{\mathbf{b}}_{q}\right) \overline{\mathbf{c}}_{r} \mathbf{d} \overline{\mathbf{b}}_{r}-2\left(\dot{\mathbf{b}}_{p} \cdot \dot{\mathbf{b}}_{r}\right) \overline{\mathbf{c}}_{r} \mathbf{d} \overline{\mathbf{b}}_{q}\right)\right\} .
$$


Proof. We have $\mathbf{a}_{i} \cdot \mathbf{a}_{j}=\sum_{p, q=1}^{n-1}\left(\mathbf{c}_{p} \cdot \mathbf{c}_{q}\right) b_{p i} b_{q j}$. So

$$
\begin{aligned}
\sum_{i, j=0}^{3}\left(\dot{\mathbf{a}}_{i} \cdot \dot{\mathbf{a}}_{j}\right)^{2} & =\sum_{i, j=0}^{3} \sum_{p, q, r, s=1}^{n-1} b_{p i} b_{q j} b_{r i} b_{s j}\left(\dot{\mathbf{c}}_{p} \cdot \dot{\mathbf{c}}_{q}\right)\left(\dot{\mathbf{c}}_{r} \cdot \dot{\mathbf{c}}_{s}\right) \\
& =\sum_{p, q, r, s=1}^{n-1}\left(\dot{\mathbf{c}}_{p} \cdot \dot{\mathbf{c}}_{q}\right)\left(\dot{\mathbf{c}}_{r} \cdot \dot{\mathbf{c}}_{s}\right)\left(\dot{\mathbf{b}}_{p} \cdot \dot{\mathbf{b}}_{r}\right)\left(\dot{\mathbf{b}}_{q} \cdot \dot{\mathbf{b}}_{s}\right) ; \\
\left(\sum_{i=0}^{3} \dot{\mathbf{a}}_{i} \cdot \mathbf{a}_{i}\right)^{2} & =\left(\sum_{i=0}^{3} \sum_{p, q, r=1}^{n-1} b_{p i} b_{q i} \dot{\mathbf{c}}_{p} \cdot \dot{\mathbf{c}}_{q}\right)^{2} \\
& =\left(\sum_{p, q=1}^{n-1}\left(\dot{\mathbf{c}}_{p} \cdot \dot{\mathbf{c}}_{q}\right)\left(\dot{\mathbf{b}}_{p} \cdot \dot{\mathbf{b}}_{q}\right)\right)^{2} ; \\
& =\sum_{p, q, r, s=1}^{n-1} b_{p 1} b_{q 2} b_{r 3} b_{s 4}\left[\dot{\mathbf{c}}_{2} \dot{\mathbf{a}}_{3} \dot{\mathbf{c}}_{q} \dot{\mathbf{c}}_{r} \dot{\mathbf{c}}_{s}\right] \\
& =\sum_{1 \leq p<q<r<s \leq n-1}\left[\dot{\mathbf{c}}_{p} \dot{\mathbf{c}}_{q} \dot{\mathbf{c}}_{r} \dot{\mathbf{c}}_{s}\right]\left[\dot{\mathbf{b}}_{p} \dot{\mathbf{b}}_{q} \dot{\mathbf{b}}_{r} \mathbf{b}_{s}\right] .
\end{aligned}
$$

Substituting them into (4.1), we get (4.10).

Consider the action of $\operatorname{adj}(\mathbf{A})$ upon $\mathbf{d}$. We divide the expression of $\operatorname{adj}(\mathbf{A})$ in (4.5) into two parts, the first part $P_{1}$ is $\left(2 \overline{\mathbf{a}}_{123}^{\sim} \mid 1\right)+\left(2 \overline{\mathbf{a}}_{023}^{\sim} \mid \mathbf{i}\right)-$ $\left(2 \overline{\mathbf{a}}_{013}^{\sim} \mid \mathbf{j}\right)+\left(2 \overline{\mathbf{a}}_{012}^{\sim} \mid \mathbf{k}\right)$, and the second part $P_{2}$ is the rest.

For $P_{1}$,

$$
\begin{aligned}
& \overline{\mathbf{a}}_{123}^{\sim}=\sum_{i=0}^{3}\left[\mathbf{e}_{i} \overline{\overline{\mathbf{a}}}_{1} \overline{\mathbf{a}}_{2} \overline{\mathbf{a}}_{3}\right] \mathbf{f}^{-1}\left(\mathbf{e}_{i}\right) \\
& =\sum_{i=0}^{3} \sum_{p, q, r=1}^{n-1}\left[\mathbf{e}_{i} \dot{\overline{\mathbf{c}}}_{p} \dot{\overline{\mathbf{c}}}_{q} \dot{\overline{\mathbf{c}}}_{r}\right] b_{p 1} b_{q 2} b_{r 3} \mathbf{f}^{-1}\left(\mathbf{e}_{i}\right) \\
& =\sum_{i=0}^{3} \sum_{1 \leq p<q<r \leq n-1}\left[\mathbf{e}_{i} \dot{\bar{c}}_{p} \dot{\mathbf{c}}_{q} \dot{\mathbf{c}}_{r}\right]\left[\mathbf{e}_{0} \dot{\mathbf{b}}_{p} \dot{\mathbf{b}}_{q} \dot{\mathbf{b}}_{r}\right] \mathbf{f}^{-1}\left(\mathbf{e}_{i}\right) .
\end{aligned}
$$

Similarly, if denoting

$$
\overline{\mathbf{a}}_{\tilde{1}}^{\sim}:=\overline{\mathbf{a}}_{023}^{\sim}, \quad \overline{\mathbf{a}}_{\tilde{2}}^{\sim}:=\overline{\mathbf{a}}_{013}^{\sim}, \quad \overline{\mathbf{a}}_{\tilde{3}}^{\sim}:=\overline{\mathbf{a}}_{012}^{\sim},
$$

then for $j=1,2,3$,

$$
\overline{\mathbf{a}}_{\tilde{j}}^{\sim}=\sum_{i=0}^{3} \sum_{1 \leq p<q<r \leq n-1}(-1)^{j-1}\left[\mathbf{e}_{i} \dot{\mathbf{c}}_{p} \dot{\mathbf{c}}_{q} \dot{\overline{\mathbf{c}}}_{r}\right]\left[\mathbf{e}_{j} \dot{\mathbf{b}}_{p} \dot{\mathbf{b}}_{q} \dot{\mathbf{b}}_{r}\right] \mathbf{f}^{-1}\left(\mathbf{e}_{i}\right) .
$$


So

$$
\begin{aligned}
& \overline{\mathbf{a}}_{123}^{\sim} \mathbf{d}+\overline{\mathbf{a}}_{023}^{\sim} \mathbf{d} \mathbf{i}-\overline{\mathbf{a}}_{013}^{\sim} \mathbf{d} \mathbf{j}+\overline{\mathbf{a}}_{012}^{\sim} \mathbf{d} \mathbf{k} \\
= & \sum_{i, j=0}^{3} \sum_{1 \leq p<q<r \leq n-1}\left[\mathbf{e}_{i} \dot{\mathbf{c}}_{p} \dot{\mathbf{c}}_{q} \overline{\mathbf{c}}_{r}\right]\left[\mathbf{e}_{j} \dot{\mathbf{b}}_{p} \dot{\mathbf{b}}_{q} \dot{\mathbf{b}}_{r}\right] \mathbf{f}^{-1}\left(\mathbf{e}_{i}\right) \mathbf{d} \overline{\mathbf{f}} \overline{\mathbf{f}}^{-1}\left(\mathbf{e}_{j}\right) \\
= & -\sum_{i, j=0}^{3} \sum_{1 \leq p<q<r \leq n-1}\left[\mathbf{e}_{i} \overline{\mathbf{c}}_{p} \overline{\mathbf{c}}_{q} \overline{\mathbf{c}}_{r}\right]\left[\mathbf{e}_{j} \overline{\mathbf{b}}_{p} \overline{\mathbf{b}}_{q} \overline{\mathbf{b}}_{r}\right] \mathbf{f}^{-1}\left(\mathbf{e}_{i}\right) \mathbf{d} \mathbf{f}^{-1}\left(\mathbf{e}_{j}\right) \\
= & -\sum_{1 \leq p<q<r \leq n-1}^{n-1} \overline{\mathbf{c}}_{p q r}^{\sim} \mathbf{d} \overline{\mathbf{b}}_{p q r}^{\sim} \\
= & -\frac{1}{6} \sum_{p, q, r=1}^{n-1} \overline{\mathbf{c}}_{p q r}^{\sim} \mathbf{d} \overline{\mathbf{b}}_{p q r}^{\sim} .
\end{aligned}
$$

For $P_{2}$, we have

$$
\begin{aligned}
& \left(2 \operatorname{adj}\left(\overline{\mathbf{a}}_{0}\right)-\Lambda \overline{\mathbf{a}}_{0}\right) \mathbf{d}-\left(2 \operatorname{adj}\left(\overline{\mathbf{a}}_{1}\right)-\Lambda \overline{\mathbf{a}}_{1}\right) \mathbf{d i} \\
& -\left(2 \operatorname{adj}\left(\overline{\mathbf{a}}_{2}\right)-\Lambda \overline{\mathbf{a}}_{2}\right) \mathbf{d j} \mathbf{j}-\left(2 \operatorname{adj}\left(\overline{\mathbf{a}}_{3}\right)-\Lambda \overline{\mathbf{a}}_{3}\right) \mathbf{d} \mathbf{k} \\
& =\sum_{p, q, r=1}^{n-1}\left(\dot{\mathbf{c}}_{p} \cdot \dot{\mathbf{c}}_{q}\right) \overline{\mathbf{c}}_{r} \mathbf{d}\left(\left(b_{p 0} b_{q 0}-b_{p 1} b_{q 1}-b_{p 2} b_{q 2}-b_{p 3} b_{q 3}\right) b_{r 0}\right. \\
& +\left(b_{p 0} b_{q 0}-b_{p 1} b_{q 1}+b_{p 2} b_{q 2}+b_{p 3} b_{q 3}\right) b_{r 1} \mathbf{i} \\
& +\left(b_{p 0} b_{q 0}+b_{p 1} b_{q 1}-b_{p 2} b_{q 2}+b_{p 3} b_{q 3}\right) b_{r 2} \mathbf{j} \\
& \left.+\left(b_{p 0} b_{q 0}+b_{p 1} b_{q 1}+b_{p 2} b_{q 2}-b_{p 3} b_{q 3}\right) b_{r 3} \mathbf{k}\right) \\
& +2 \sum_{p, q, r=1}^{n-1}\left(\dot{\mathbf{c}}_{p} \cdot \dot{\mathbf{c}}_{q}\right) \overline{\mathbf{c}}_{r} \mathbf{d}\left(b_{p 0} b_{q 0} b_{r 0}-b_{p 0} b_{r 0}\left(b_{q 1} \mathbf{i}+b_{q 2} \mathbf{j}+b_{q 3} \mathbf{k}\right)\right. \\
& +b_{p 0} b_{q 1} b_{r 1}-b_{p 1} b_{q 1} b_{r 1} \mathbf{i}-b_{p 1} b_{q 2} b_{r 1} \mathbf{j}-b_{p 1} b_{q 3} b_{r 1} \mathbf{k} \\
& +b_{p 0} b_{q 2} b_{r 2}-b_{p 1} b_{q 2} b_{r 2} \mathbf{i}-b_{p 2} b_{q 2} b_{r 2} \mathbf{j}-b_{p 2} b_{q 3} b_{r 2} \mathbf{k} \\
& \left.+b_{p 0} b_{q 3} b_{r 3}-b_{p 1} b_{q 3} b_{r 3} \mathbf{i}-b_{p 2} b_{q 3} b_{r 3} \mathbf{j}-b_{p 3} b_{q 3} b_{r 3} \mathbf{k}\right) \\
& =-\sum_{p, q, r=1}^{n-1}\left(\mathbf{c}_{p} \cdot \mathbf{c}_{q}\right)\left(\mathbf{b}_{p} \cdot \mathbf{b}_{q}\right) \overline{\mathbf{c}}_{r} \mathbf{d} \overline{\mathbf{b}}_{r}+2 \sum_{p, q, r=1}^{n-1}\left(\mathbf{c}_{p} \cdot \mathbf{c}_{q}\right)\left(\mathbf{b}_{p} \cdot \mathbf{b}_{r}\right) \overline{\mathbf{c}}_{r} \mathbf{d} \overline{\mathbf{b}}_{q} .
\end{aligned}
$$

Combining the results of $P_{1}, P_{2}$, we get (4.11).

Remark: the solution (4.11) is just the following identity in $\mathbb{H}$ :

$$
\begin{aligned}
& \sum_{p, q, r=1}^{n-1}\left\{\left(\overline{\mathbf{c}}_{p q r}^{\sim} \mid \overline{\mathbf{b}}_{p q r}^{\sim}\right)+3\left(\dot{\mathbf{c}}_{p} \cdot \dot{\mathbf{c}}_{q}\right)\left(\left(\dot{\mathbf{b}}_{p} \cdot \dot{\mathbf{b}}_{q}\right)\left(\overline{\mathbf{c}}_{r} \mid \overline{\mathbf{b}}_{r}\right)\right.\right. \\
& \left.\left.-2\left(\dot{\mathbf{b}}_{p} \cdot \dot{\mathbf{b}}_{r}\right)\left(\overline{\mathbf{c}}_{r} \mid \overline{\mathbf{b}}_{q}\right)\right)\right\}\left(\sum_{s=1}^{n-1}\left(\mathbf{c}_{s} \mid \mathbf{b}_{s}\right)\right) \\
& =-3 \operatorname{det}\left(\sum_{p=1}^{n-1}\left(\mathbf{c}_{p} \mid \mathbf{b}_{p}\right)\right)(1 \mid 1) .
\end{aligned}
$$




\section{The Clifford algebraic approach}

Assume that the input linear quaternionic equation (1.1) is the image of an equation in $C L\left(\mathbb{R}^{4}\right)$ under the projection $\pi$, where the preimages of the $\mathbf{c}_{i}, \mathbf{q}, \mathbf{b}_{i}, \mathbf{d}$ under $\pi$ are vectors of $\mathbb{R}^{4}$. The corresponding equation in $C L\left(\mathbb{R}^{4}\right)$ is called the lift of the quaternionic equation.

Lemma 5.1. For any $\mathbf{b}_{i}, \mathbf{c}_{i}, \mathbf{d}, \mathbf{q} \in \mathbb{H}, \sum_{i=1}^{n-1} \mathbf{c}_{i} \mathbf{q} \mathbf{b}_{i}-\mathbf{d}=0$ if and only if

$$
\left(\sum_{i=1}^{n-1} \dot{\mathbf{c}}_{i} \overline{\mathbf{q}}^{\prime} \dot{\mathbf{b}}_{i}-\mathbf{d}\right)\left(1+\mathbf{I}_{4}\right)=0 \text {. }
$$

Proof. Obviously $\pi\left(\left(\sum_{i=1}^{n-1} \mathbf{c}_{i} \overline{\mathbf{q}}^{\prime} \mathbf{b}_{i}-\mathbf{d}\right)\left(1+\mathbf{I}_{4}\right)\right)=\left(\sum_{i=1}^{n-1} \mathbf{c}_{i} \mathbf{q} \mathbf{b}_{i}-\mathbf{d}\right)(1+$ $\left.\pi\left(\overline{\mathbf{I}_{4}}\right)\right)=2\left(\sum_{i=1}^{n-1} \mathbf{c}_{i} \mathbf{q} \mathbf{b}_{i}-\mathbf{d}\right)$. So (5.1) leads to the input equation (1.1).

Conversely, by (3.13), for an $\mathbf{A} \in K_{-}$(the odd elements of $k e r(\pi)$ ), there exists an $\mathbf{x} \in \mathbb{R}^{4}$ such that $\mathbf{A}=\mathbf{x}\left(1-\mathbf{I}_{4}\right)$. Since $\pi\left(\sum_{i=1}^{n-1} \dot{\mathbf{c}}_{i} \overline{\mathbf{q}}^{\prime} \mathbf{b}_{i}-\mathbf{d}\right)=$ $\sum_{i=1}^{n-1} \mathbf{c}_{i} \mathbf{q} \mathbf{b}_{i}-\mathbf{d}=0$, there exists $\mathbf{x} \in \mathbb{R}^{4}$ such that

$$
\sum_{i=1}^{n-1} \mathbf{c}_{i} \overline{\mathbf{q}}^{\prime} \dot{b}_{i}-\mathbf{d}=\mathbf{x}\left(1-\mathbf{I}_{4}\right)
$$

Multiplying both sides from the right by $1+\mathbf{I}_{4}$, and using $\left(1-\mathbf{I}_{4}\right)\left(1+\mathbf{I}_{4}\right)=$ $1-\mathbf{I}_{4}^{2}=0$, we get (5.1).

Below we solve (5.1) for vector variable $\overline{\mathbf{q}}$.

Let $I_{\text {init }}$ be the two-sided ideal generated by $\sum_{i=1}^{n-1} \mathbf{c}_{i} \mathbf{q} \mathbf{b}_{i}-\mathbf{d} \in \mathbb{H}$. Let $I_{+}$be the $\mathbb{R}$-linear combination of the following elements in $C L\left(\mathbb{R}^{4}\right)$ :

$$
\mathbf{B}\left(\sum_{i=1}^{n-1} \dot{\mathbf{c}}_{i} \overline{\mathbf{q}} \mathbf{b}_{i}-\mathbf{d}\right)\left(1+\mathbf{I}_{4}\right) \mathbf{C}
$$

where $\mathbf{B}, \mathbf{C} \in C L\left(\mathbb{R}^{4}\right)$ and $\mathbf{B}$ is an even element. $I_{+}$is called the left-even two-sided ideal generated by $\left(\sum_{i=1}^{n-1} \mathbf{c}_{i} \overline{\mathbf{q}}^{\prime} \mathbf{b}_{i}-\mathbf{d}\right)\left(1+\mathbf{I}_{4}\right)$.

Lemma 5.2. $\pi\left(I_{+}\right) \subset I_{\text {init }}$.

Proof. The result follows

$$
\pi\left(\mathbf{B}\left(\sum_{i=1}^{n-1} \dot{\mathbf{c}}_{i} \overline{\mathbf{q}}^{\prime} \dot{\mathbf{b}}_{i}-\mathbf{d}\right)\left(1+\mathbf{I}_{4}\right) \mathbf{C}\right)=2 \pi(\mathbf{B})\left(\sum_{i=1}^{n-1} \mathbf{c}_{i} \mathbf{q} \mathbf{b}_{i}-\mathbf{d}\right) \pi(\overline{\mathbf{C}}) \in I_{\text {init }} .
$$

So if $\mathbf{A} \in C L\left(\mathbb{R}^{4}\right)$ is an odd element, then $\mathbf{e}_{0} \mathbf{A}\left(\sum_{i=1}^{n-1} \dot{\mathbf{c}}_{i} \overline{\mathbf{q}}^{\prime} \mathbf{b}_{i}-\mathbf{d}\right)(1+$ $\left.\mathbf{I}_{4}\right) \mathbf{C} \in I_{+}$, and $\pi\left(\mathbf{e}_{0} \mathbf{A}\left(\sum_{i=1}^{n-1} \dot{\mathbf{c}}_{i} \overline{\mathbf{q}}^{\prime} \mathbf{b}_{i}-\mathbf{d}\right)\left(1+\mathbf{I}_{4}\right) \mathbf{C}\right) \in I_{\text {init }}$.

Notation. For $\mathbf{b}_{i}, \mathbf{c}_{j} \in \mathbb{H}$, denote

$$
\begin{array}{ll}
\hat{\mathbf{b}}_{i j}:=\dot{\mathbf{b}}_{i} \wedge \dot{\mathbf{b}}_{j}, & \dot{\mathbf{b}}_{i j k}:=\dot{\mathbf{b}}_{i} \wedge \dot{\mathbf{b}}_{j} \wedge \dot{\mathbf{b}}_{k}, \\
\dot{\mathbf{c}}_{i j}:=\dot{\mathbf{c}}_{i} \wedge \dot{\mathbf{c}}_{j}, & \dot{\mathbf{c}}_{i j k}:=\dot{\mathbf{c}}_{i} \wedge \dot{\mathbf{c}}_{j} \wedge \dot{\mathbf{c}}_{k} .
\end{array}
$$


Applying the linear operator $\sum_{p, q, r=1}^{n-1}\left(\mathbf{e}_{0} \dot{\mathbf{c}}_{p q r}^{\sim} \mid \hat{\mathbf{b}}_{p q r}^{\sim}\right)$ to both sides of (5.1), we get

$$
\sum_{i, p, q, r=1}^{n-1} \mathbf{e}_{0} \dot{\mathbf{c}}_{p q r}^{\sim} \dot{\mathbf{c}}_{i} \overline{\mathbf{q}} \mathbf{b}_{i} \dot{\mathbf{b}}_{p q r}^{\sim}\left(1-\mathbf{I}_{4}\right)=\sum_{p, q, r=1}^{n-1} \mathbf{e}_{0} \dot{\mathbf{c}}_{p q r}^{\sim} \dot{\mathbf{d}} \dot{\mathbf{b}}_{p q r}^{\sim}\left(1-\mathbf{I}_{4}\right) .
$$

Lemma 5.3. 4] For any $\mathbf{x}_{l} \in \mathbb{R}^{4}$,

$$
\begin{aligned}
\mathbf{x}_{1} \mathbf{x}_{2}= & \mathbf{x}_{1} \cdot \mathbf{x}_{2}+\mathbf{x}_{1} \wedge \mathbf{x}_{2}, \\
\mathbf{x}_{1}\left(\mathbf{x}_{2} \wedge \mathbf{x}_{3} \wedge \mathbf{x}_{4}\right)^{\sim}= & {\left[\mathbf{x}_{1} \mathbf{x}_{2} \mathbf{x}_{3} \mathbf{x}_{4}\right]+\left(\mathbf{x}_{1} \cdot \mathbf{x}_{2}\right)\left(\mathbf{x}_{3} \wedge \mathbf{x}_{4}\right)^{\sim} } \\
& -\left(\mathbf{x}_{1} \cdot \mathbf{x}_{3}\right)\left(\mathbf{x}_{2} \wedge \mathbf{x}_{4}\right)^{\sim}+\left(\mathbf{x}_{1} \cdot \mathbf{x}_{4}\right)\left(\mathbf{x}_{1} \wedge \mathbf{x}_{4}\right)^{\sim} \\
\left(\mathbf{x}_{2} \wedge \mathbf{x}_{3} \wedge \mathbf{x}_{4}\right)^{\sim} \mathbf{x}_{1}= & {\left[\mathbf{x}_{1} \mathbf{x}_{2} \mathbf{x}_{3} \mathbf{x}_{4}\right]-\left(\mathbf{x}_{1} \cdot \mathbf{x}_{2}\right)\left(\mathbf{x}_{3} \wedge \mathbf{x}_{4}\right)^{\sim} } \\
& +\left(\mathbf{x}_{1} \cdot \mathbf{x}_{3}\right)\left(\mathbf{x}_{2} \wedge \mathbf{x}_{4}\right)^{\sim}-\left(\mathbf{x}_{1} \cdot \mathbf{x}_{4}\right)\left(\mathbf{x}_{1} \wedge \mathbf{x}_{4}\right)^{\sim} .
\end{aligned}
$$

Applying Lemma 5.3 to the left side of (5.5), we get

$$
\begin{aligned}
& \text { L.H.S. } \\
& =\sum_{i, p, q, r=1}^{n-1} \mathbf{e}_{0}\left(\left[\dot{\mathbf{c}}_{i} \dot{\mathbf{c}}_{p} \dot{\mathbf{c}}_{q} \dot{\mathbf{c}}_{r}\right]-\left(\left(\dot{\mathbf{c}}_{i} \cdot \dot{\mathbf{c}}_{p}\right) \dot{\mathbf{c}}_{q r}-\left(\dot{\mathbf{c}}_{i} \cdot \dot{\mathbf{c}}_{q}\right) \dot{\mathbf{c}}_{p r}+\left(\dot{\mathbf{c}}_{i} \cdot \dot{\mathbf{c}}_{r}\right) \mathbf{c}_{p q}\right) \sim\right) \overline{\mathbf{q}} \\
& \left(\left[\dot{\mathbf{b}}_{i} \dot{\mathbf{b}}_{p} \dot{\mathbf{b}}_{q} \dot{\mathbf{b}}_{r}\right]+\left(\left(\dot{\mathbf{b}}_{i} \cdot \dot{\mathbf{b}}_{p}\right) \dot{\mathbf{b}}_{q r}-\left(\dot{\mathbf{b}}_{i} \cdot \dot{\mathbf{b}}_{q}\right) \dot{\mathbf{c}}_{p r}+\left(\dot{\mathbf{b}}_{i} \cdot \dot{\mathbf{b}}_{r}\right) \dot{\mathbf{b}}_{p q}\right)^{\sim}\right)\left(1-\mathbf{I}_{4}\right) \\
& :=\Sigma_{1}+\Sigma_{2}-\Sigma_{3}-\Sigma_{4}
\end{aligned}
$$

where

$$
\begin{aligned}
& \Sigma_{1}=\sum_{i, p, q, r=1}^{n-1}\left[\dot{c}_{i} \dot{\mathbf{c}}_{p} \dot{\mathbf{c}}_{q} \dot{\mathbf{c}}_{r}\right]\left[\dot{\mathbf{b}}_{i} \dot{\mathbf{b}}_{p} \dot{\mathbf{b}}_{q} \dot{\mathbf{b}}_{r}\right] \mathbf{e}_{0} \overline{\mathbf{q}}\left(1-\mathbf{I}_{4}\right) \\
& \Sigma_{2}=\sum_{i, p, q, r=1}^{n-1}\left[\dot{c}_{i} \dot{\mathbf{c}}_{p} \mathbf{c}_{q} \dot{\mathbf{c}}_{r}\right] \mathbf{e}_{0} \overline{\mathbf{q}}\left(\left(\mathbf{b}_{i} \cdot \hat{\mathbf{b}}_{p}\right) \mathbf{b}_{q r}-\left(\hat{\mathbf{b}}_{i} \cdot \mathbf{b}_{q}\right) \mathbf{b}_{p r}+\left(\hat{\mathbf{b}}_{i} \cdot \mathbf{b}_{r}\right) \mathbf{b}_{p q}\right)\left(\mathbf{I}_{4}-1\right) ; \\
& \Sigma_{3}=\sum_{i, p, q, r=1}^{n-1}\left[\dot{\mathbf{b}}_{i} \dot{\mathbf{b}}_{p} \dot{\mathbf{b}}_{q} \dot{\mathbf{b}}_{r}\right] \mathbf{e}_{0}\left(\left(\dot{\mathbf{c}}_{i} \cdot \dot{\mathbf{c}}_{p}\right) \dot{\mathbf{c}}_{q r}-\left(\dot{\mathbf{c}}_{i} \cdot \dot{\mathbf{c}}_{q}\right) \dot{\mathbf{c}}_{p r}+\left(\dot{\mathbf{c}}_{i} \cdot \dot{\mathbf{c}}_{r}\right) \dot{\mathbf{c}}_{p q}\right) \overline{\mathbf{q}}\left(1-\mathbf{I}_{4}\right) \\
& \Sigma_{4}=\sum_{i, p, q, r=1}^{n-1} \mathbf{e}_{0}\left(\left(\dot{\mathbf{c}}_{i} \cdot \dot{\mathbf{c}}_{p}\right) \dot{\mathbf{c}}_{q r}-\left(\dot{\mathbf{c}}_{i} \cdot \dot{\mathbf{c}}_{q}\right) \dot{\mathbf{c}}_{p r}+\left(\dot{\mathbf{c}}_{i} \cdot \dot{\mathbf{c}}_{r}\right) \dot{\mathbf{c}}_{p q}\right) \overline{\mathbf{q}} \\
& \left(\left(\mathbf{b}_{i} \cdot \mathbf{b}_{p}\right) \mathbf{b}_{q r}-\left(\mathbf{b}_{i} \cdot \mathbf{b}_{q}\right) \mathbf{b}_{p r}+\left(\mathbf{b}_{i} \cdot \mathbf{b}_{r}\right) \mathbf{b}_{p q}\right)\left(\mathbf{I}_{4}-1\right) .
\end{aligned}
$$

By the symmetry of the inner product and the antisymmetry of the outer product, $\Sigma_{2}=\Sigma_{3}=0$. For $\Sigma_{4}$, again by the two symmetries,

$\Sigma_{4}=\sum_{i, p, q, r=1}^{n-1}\left(\dot{\mathbf{c}}_{i} \cdot \dot{\mathbf{c}}_{p}\right)\left(-3\left(\dot{\mathbf{b}}_{i} \cdot \dot{\mathbf{b}}_{p}\right) \mathbf{e}_{0} \dot{\mathbf{c}}_{q r} \overline{\mathbf{q}} \dot{b}_{q r}+6\left(\dot{\mathbf{b}}_{i} \cdot \dot{\mathbf{b}}_{q}\right) \mathbf{e}_{0} \dot{\mathbf{c}}_{q r} \overline{\mathbf{q}} \dot{b}_{p r}\right)\left(1-\mathbf{I}_{4}\right)$.

Thus we get the following result: 
Lemma 5.4. The following element is in $I_{+}$:

$$
\begin{aligned}
& \left(-\sum_{p, q, r=1}^{n-1} \mathbf{e}_{0} \dot{\mathbf{c}}_{p q r}^{\sim} \mathbf{d} \mathbf{b} \hat{\mathbf{b}}_{p q r}^{\sim}+\sum_{i, p, q, r=1}^{n-1}\left[\dot{c}_{i} \dot{\mathbf{c}}_{p} \dot{\mathbf{c}}_{q} \dot{\mathbf{c}}_{r}\right]\left[\dot{\mathbf{b}}_{i} \dot{\mathbf{b}}_{p} \dot{\mathbf{b}}_{q} \mathbf{b}_{r}\right] \mathbf{e}_{0} \overline{\mathbf{q}}\right. \\
& \left.+\sum_{i, p, q, r=1}^{n-1}\left(\dot{\mathbf{c}}_{i} \cdot \dot{\mathbf{c}}_{p}\right)\left(6\left(\dot{\mathbf{b}}_{i} \cdot \dot{\mathbf{b}}_{q}\right) \mathbf{e}_{0} \dot{\mathbf{c}}_{q r} \overline{\mathbf{q}} \dot{\mathbf{b}}_{p r}-3\left(\dot{\mathbf{b}}_{i} \cdot \dot{\mathbf{b}}_{p}\right) \mathbf{e}_{0} \dot{\mathbf{c}}_{q r} \overline{\mathbf{q}} \dot{\mathbf{b}}_{q r}\right)\right)\left(1-\mathbf{I}_{4}\right) \text {. }
\end{aligned}
$$

In (5.9), the last summation can be generated by applying two linear operators to the left side of equation (5.1) respectively: applying linear operator $\sum_{i, p, q=1}^{n-1}\left(\dot{\mathbf{c}}_{i} \cdot \mathbf{c}_{p}\right)\left(\dot{\mathbf{b}}_{i} \cdot \mathbf{b}_{q}\right)\left(\mathbf{e}_{0} \dot{\mathbf{c}}_{q} \mid \dot{\mathbf{b}}_{p}\right)$ to (5.1), we get

$$
\begin{aligned}
\sum_{i, p, q, r=1}^{n-1}\left(\dot{\mathbf{c}}_{i} \cdot \dot{\mathbf{c}}_{p}\right)\left(\dot{\mathbf{b}}_{i} \cdot \mathbf{b}_{q}\right) \mathbf{e}_{0} \dot{\mathbf{c}}_{q} \mathbf{c}_{r} \overline{\mathbf{q}} \mathbf{b}_{r} \mathbf{b}_{p}\left(1-\mathbf{I}_{4}\right) \\
=\sum_{i, p, q=1}^{n-1}\left(\dot{\mathbf{c}}_{i} \cdot \dot{\mathbf{c}}_{p}\right)\left(\dot{\mathbf{b}}_{i} \cdot \dot{\mathbf{b}}_{q}\right) \mathbf{e}_{0} \dot{\mathbf{c}}_{q} \mathbf{d} \mathbf{b} \dot{b}_{p}\left(1-\mathbf{I}_{4}\right)
\end{aligned}
$$

applying $\sum_{i, p, q=1}^{n-1}\left(\dot{\mathbf{c}}_{i} \cdot \dot{\mathbf{c}}_{p}\right)\left(\mathbf{b}_{i} \cdot \mathbf{b}_{p}\right)\left(\mathbf{e}_{0} \dot{\mathbf{c}}_{q} \mid \mathbf{b}_{q}\right)$ to (5.1), we get

$$
\begin{aligned}
\sum_{i, p, q, r=1}^{n-1}\left(\dot{\mathbf{c}}_{i} \cdot \dot{\mathbf{c}}_{p}\right)\left(\dot{\mathbf{b}}_{i} \cdot \dot{\mathbf{b}}_{p}\right) \mathbf{e}_{0} \dot{\mathbf{c}}_{q} \dot{\mathbf{c}}_{r} \overline{\mathbf{q}} \dot{\mathbf{b}}_{r} \dot{\mathbf{b}}_{q}\left(1-\mathbf{I}_{4}\right) \\
=\sum_{i, p, q=1}^{n-1}\left(\dot{\mathbf{c}}_{i} \cdot \dot{\mathbf{c}}_{p}\right)\left(\dot{\mathbf{b}}_{i} \cdot \dot{\mathbf{b}}_{p}\right) \dot{\mathbf{c}}_{q} \dot{\mathbf{d}} \dot{\mathbf{b}}_{q}\left(1-\mathbf{I}_{4}\right) .
\end{aligned}
$$

Lemma 5.5. The following two elements are in $I_{+}$:

$$
\begin{aligned}
& \left(-\sum_{i, p, q=1}^{n-1}\left(\dot{\mathbf{c}}_{i} \cdot \dot{\mathbf{c}}_{p}\right)\left(\mathbf{b}_{i} \cdot \dot{\mathbf{b}}_{q}\right) \mathbf{e}_{0} \dot{\mathbf{c}}_{q} \mathbf{d} \mathbf{b}_{p}\right. \\
& +\sum_{i, p, q, r=1}^{n-1}\left(\dot{\mathbf{c}}_{i} \cdot \dot{\mathbf{c}}_{p}\right)\left(\dot{\mathbf{b}}_{i} \cdot \dot{\mathbf{b}}_{q}\right)\left(\dot{\mathbf{c}}_{q} \cdot \dot{\mathbf{c}}_{r}\right)\left(\dot{\mathbf{b}}_{p} \cdot \dot{\mathbf{b}}_{r}\right) \mathbf{e}_{0} \overline{\mathbf{q}} \\
& \left.-\sum_{i, p, q, r=1}^{n-1}\left(\dot{\mathbf{c}}_{i} \cdot \dot{\mathbf{c}}_{p}\right)\left(\dot{\mathbf{b}}_{i} \cdot \dot{\mathbf{b}}_{q}\right) \mathbf{e}_{0} \dot{\mathbf{c}}_{q r} \mathbf{q}^{\prime} \dot{\mathbf{b}}_{p r}\right)\left(1-\mathbf{I}_{4}\right) ; \\
& \left(-\sum_{i, p, q=1}^{n-1}\left(\dot{\mathbf{c}}_{i} \cdot \dot{\mathbf{c}}_{p}\right)\left(\dot{\mathbf{b}}_{i} \cdot \mathbf{b}_{p}\right) \mathbf{e}_{0} \dot{\mathbf{c}}_{q} \mathbf{d} \mathbf{b}_{q}\right. \\
& +\sum_{i, p, q, r=1}^{n-1}\left(\dot{\mathbf{c}}_{i} \cdot \dot{\mathbf{c}}_{p}\right)\left(\dot{\mathbf{b}}_{i} \cdot \dot{\mathbf{b}}_{p}\right)\left(\dot{\mathbf{c}}_{q} \cdot \dot{\mathbf{c}}_{r}\right)\left(\dot{\mathbf{b}}_{q} \cdot \dot{\mathbf{b}}_{r}\right) \mathbf{e}_{0} \overline{\mathbf{q}} \\
& \left.-\sum_{i, p, q, r=1}^{n-1}\left(\dot{\mathbf{c}}_{i} \cdot \dot{\mathbf{c}}_{p}\right)\left(\dot{\mathbf{b}}_{i} \cdot \dot{\mathbf{b}}_{p}\right) \mathbf{e}_{0} \dot{\mathbf{c}}_{q r} \dot{\mathbf{q}}^{\prime} \mathbf{b}_{q r}\right)\left(1-\mathbf{I}_{4}\right) \text {. }
\end{aligned}
$$


Proof. Applying Lemma 5.3 to the left side of (5.10), then by the symmetry of the inner product and the antisymmetry of the outer product, we get

$$
\begin{aligned}
& \text { L.H.S. }=\sum_{i, p, q, r=1}^{n-1}\left(\dot{\mathbf{c}}_{i} \cdot \dot{\mathbf{c}}_{p}\right)\left(\dot{\mathbf{b}}_{i} \cdot \dot{\mathbf{b}}_{q}\right)\left(\dot{\mathbf{c}}_{q} \cdot \dot{\mathbf{c}}_{r}+\dot{\mathbf{c}}_{q r}\right) \mathbf{e}_{0} \overline{\mathbf{q}}\left(\dot{\mathbf{b}}_{r} \cdot \dot{\mathbf{b}}_{p}-\dot{\mathbf{b}}_{p r}\right)\left(1-\mathbf{I}_{4}\right) \\
& =\sum_{i, p, q, r=1}^{n-1}\left(\dot{\mathbf{c}}_{i} \cdot \dot{\mathbf{c}}_{p}\right)\left(\dot{\mathbf{b}}_{i} \cdot \dot{\mathbf{b}}_{q}\right)\left(\dot{\mathbf{c}}_{q} \cdot \dot{\mathbf{c}}_{r}\right)\left(\dot{\mathbf{b}}_{r} \cdot \dot{\mathbf{b}}_{p}\right) \mathbf{e}_{0} \overline{\mathbf{q}}\left(1-\mathbf{I}_{4}\right) \\
& -\sum_{i, p, q, r=1}^{n-1}\left(\dot{\mathbf{c}}_{i} \cdot \dot{\mathbf{c}}_{p}\right)\left(\mathbf{b}_{i} \cdot \dot{\mathbf{b}}_{q}\right)\left(\dot{\mathbf{c}}_{q} \cdot \dot{\mathbf{c}}_{r}\right) \mathbf{e}_{0} \overline{\mathbf{q}} \dot{b}_{p r}\left(1-\mathbf{I}_{4}\right) \\
& +\sum_{i, p, q, r=1}^{n-1}\left(\dot{\mathbf{c}}_{i} \cdot \dot{\mathbf{c}}_{p}\right)\left(\dot{\mathbf{b}}_{i} \cdot \dot{\mathbf{b}}_{q}\right)\left(\dot{\mathbf{b}}_{r} \cdot \dot{\mathbf{b}}_{p}\right) \mathbf{e}_{0} \dot{\mathbf{c}}_{q r} \overline{\mathbf{q}}\left(1-\mathbf{I}_{4}\right) \\
& -\sum_{i, p, q, r=1}^{n-1}\left(\dot{\mathbf{c}}_{i} \cdot \dot{\mathbf{c}}_{p}\right)\left(\dot{\mathbf{b}}_{i} \cdot \dot{\mathbf{b}}_{q}\right) \mathbf{e}_{0} \dot{\mathbf{c}}_{q r} \overline{\mathbf{q}} \mathbf{b}_{p r}\left(1-\mathbf{I}_{4}\right) \\
& =\sum_{i, p, q, r=1}^{n-1}\left(\dot{\mathbf{c}}_{i} \cdot \dot{\mathbf{c}}_{p}\right)\left(\dot{\mathbf{b}}_{i} \cdot \dot{\mathbf{b}}_{q}\right)\left(\dot{\mathbf{c}}_{q} \cdot \dot{\mathbf{c}}_{r}\right)\left(\dot{\mathbf{b}}_{r} \cdot \dot{\mathbf{b}}_{p}\right) \mathbf{e}_{0} \overline{\mathbf{q}}\left(1-\mathbf{I}_{4}\right) \\
& -\sum_{i, p, q, r=1}^{n-1}\left(\dot{\mathbf{c}}_{i} \cdot \dot{\mathbf{c}}_{p}\right)\left(\dot{\mathbf{b}}_{i} \cdot \dot{\mathbf{b}}_{q}\right) \mathbf{e}_{0} \mathbf{c}_{q r} \overline{\mathbf{q}} \dot{b}_{p r}\left(1-\mathbf{I}_{4}\right) .
\end{aligned}
$$

From the left side of (5.11), we get a similar expression. They lead to the results of the lemma.

By the above lemmas, we get

Proposition 5.6. The following element is in $I_{+}$:

$$
\begin{aligned}
& \sum_{p, q, r, s=1}^{n-1}\left\{\left[\dot{\mathbf{c}}_{p} \dot{\mathbf{c}}_{q} \dot{\mathbf{c}}_{r} \dot{\mathbf{c}}_{s}\right]\left[\dot{\mathbf{b}}_{p} \dot{\mathbf{b}}_{q} \dot{\mathbf{b}}_{r} \dot{\mathbf{b}}_{s}\right]+3\left(\dot{\mathbf{c}}_{p} \cdot \dot{\mathbf{c}}_{q}\right)\left(\dot{\mathbf{c}}_{r} \cdot \dot{\mathbf{c}}_{s}\right)\left(\dot{\mathbf{b}}_{p} \cdot \dot{\mathbf{b}}_{q}\right)\left(\dot{\mathbf{b}}_{r} \cdot \dot{\mathbf{b}}_{s}\right)\right. \\
& \left.-6\left(\dot{\mathbf{c}}_{p} \cdot \dot{\mathbf{c}}_{q}\right)\left(\dot{\mathbf{c}}_{r} \cdot \dot{\mathbf{c}}_{s}\right)\left(\dot{\mathbf{b}}_{q} \cdot \dot{\mathbf{b}}_{s}\right)\left(\dot{\mathbf{b}}_{p} \cdot \dot{\mathbf{b}}_{r}\right)\right\} \mathbf{e}_{0} \overline{\mathbf{q}}\left(1-\mathbf{I}_{4}\right) \\
& -\sum_{p, q, r=1}^{n-1}\left\{\mathbf{e}_{0} \dot{\mathbf{c}}_{p q r}^{\sim} \mathbf{d} \dot{\mathbf{b}}_{p q r}^{\sim}+3\left(\dot{\mathbf{c}}_{p} \cdot \dot{\mathbf{c}}_{r}\right)\left(\dot{\mathbf{b}}_{p} \cdot \dot{\mathbf{b}}_{r}\right) \mathbf{e}_{0} \dot{\mathbf{c}}_{q} \mathbf{d}^{\prime} \dot{b}_{q}\right. \\
& \left.-6\left(\dot{\mathbf{c}}_{p} \cdot \dot{\mathbf{c}}_{r}\right)\left(\dot{\mathbf{b}}_{q} \cdot \dot{\mathbf{b}}_{r}\right) \mathbf{e}_{0} \dot{\mathbf{c}}_{q} \mathbf{d} \mathbf{b}_{p}\right\}\left(1-\mathbf{I}_{4}\right) .
\end{aligned}
$$

Corollary 5.7. The following element is in $I_{\text {init }}$ : 


$$
\begin{array}{r}
\sum_{p, q, r, s=1}^{n-1}\left\{\left[\dot{\mathbf{c}}_{p} \dot{\mathbf{c}}_{q} \dot{\mathbf{c}}_{r} \dot{\mathbf{c}}_{s}\right]\left[\dot{\mathbf{b}}_{p} \dot{\mathbf{b}}_{q} \dot{\mathbf{b}}_{r} \dot{\mathbf{b}}_{s}\right]+3\left(\dot{\mathbf{c}}_{p} \cdot \dot{\mathbf{c}}_{q}\right)\left(\dot{\mathbf{c}}_{r} \cdot \dot{\mathbf{c}}_{s}\right)\left(\dot{\mathbf{b}}_{p} \cdot \dot{\mathbf{b}}_{q}\right)\left(\dot{\mathbf{b}}_{r} \cdot \dot{\mathbf{b}}_{s}\right)\right. \\
\left.-6\left(\dot{\mathbf{c}}_{p} \cdot \dot{\mathbf{c}}_{q}\right)\left(\dot{\mathbf{c}}_{r} \cdot \dot{\mathbf{c}}_{s}\right)\left(\dot{\mathbf{b}}_{q} \cdot \dot{\mathbf{b}}_{s}\right)\left(\dot{\mathbf{b}}_{p} \cdot \dot{\mathbf{b}}_{r}\right)\right\} \mathbf{q} \\
-\sum_{p, q, r=1}^{n-1}\left\{\overline{\mathbf{c}}_{p q r}^{\sim} \mathbf{d} \overline{\mathbf{b}}_{p q r}^{\sim}+3\left(\dot{\mathbf{c}}_{p} \cdot \dot{\mathbf{c}}_{r}\right)\left(\dot{\mathbf{b}}_{p} \cdot \dot{\mathbf{b}}_{r}\right) \overline{\mathbf{c}}_{q} \mathbf{d} \overline{\mathbf{b}}_{q}-6\left(\dot{\mathbf{c}}_{p} \cdot \dot{\mathbf{c}}_{r}\right)\left(\dot{\mathbf{b}}_{q} \cdot \dot{\mathbf{b}}_{r}\right) \overline{\mathbf{c}}_{q} \mathbf{d} \overline{\mathbf{b}}_{p}\right\} .
\end{array}
$$

It leads to Theorem 4.3 directly.

Set

$$
\begin{array}{r}
\Delta:=\sum_{p, q, r, s=1}^{n-1}\left\{\left[\dot{\mathbf{c}}_{p} \dot{\mathbf{c}}_{q} \dot{\mathbf{c}}_{r} \dot{\mathbf{c}}_{s}\right]\left[\dot{\mathbf{b}}_{p} \dot{\mathbf{b}}_{q} \dot{\mathbf{b}}_{r} \dot{\mathbf{b}}_{s}\right]+3\left(\dot{\mathbf{c}}_{p} \cdot \dot{\mathbf{c}}_{q}\right)\left(\dot{\mathbf{c}}_{r} \cdot \dot{\mathbf{c}}_{s}\right)\left(\dot{\mathbf{b}}_{p} \cdot \dot{\mathbf{b}}_{q}\right)\left(\dot{\mathbf{b}}_{r} \cdot \dot{\mathbf{b}}_{s}\right)\right. \\
\left.-6\left(\dot{\mathbf{c}}_{p} \cdot \dot{\mathbf{c}}_{q}\right)\left(\dot{\mathbf{c}}_{r} \cdot \dot{\mathbf{c}}_{s}\right)\left(\dot{\mathbf{b}}_{q} \cdot \dot{\mathbf{b}}_{s}\right)\left(\dot{\mathbf{b}}_{p} \cdot \dot{\mathbf{b}}_{r}\right)\right\} \\
\Phi:=\sum_{p, q, r=1}^{n-1}\left\{\left(\overline{\mathbf{c}}_{p q r}^{\sim} \mid \overline{\mathbf{b}}_{p q r}^{\sim}\right)+3\left(\dot{\mathbf{c}}_{p} \cdot \dot{\mathbf{c}}_{r}\right)\left(\dot{\mathbf{b}}_{p} \cdot \dot{\mathbf{b}}_{r}\right)\left(\overline{\mathbf{c}}_{q} \mid \overline{\mathbf{b}}_{q}\right)\right. \\
\left.-6\left(\dot{\mathbf{c}}_{p} \cdot \dot{\mathbf{c}}_{r}\right)\left(\dot{\mathbf{b}}_{q} \cdot \dot{\mathbf{b}}_{r}\right)\left(\overline{\mathbf{c}}_{q} \mid \overline{\mathbf{b}}_{p}\right)\right\} .
\end{array}
$$

Then $\Delta \in \mathbb{R}$, and $\Phi$ is a linear operator in $\mathbb{H}$. Let $\mathbf{A q}=\mathbf{d}$ be the associated real linear system of the input quaternionic equation, then by (4.10), $\Delta=$ $-3 \operatorname{det}(\mathbf{A})$. By Corollary 5.7. $\Phi \mathbf{A q}=\Phi \mathbf{d}=\Delta \mathbf{q}=-3 \operatorname{det}(\mathbf{A}) \mathbf{q}$. So in matrix form,

$$
\Phi=-3 \operatorname{adj}(\mathbf{A})
$$

The non-degeneracy of $\Phi$ is the same with that of $\mathbf{A}$.

\section{General linear quaternionic equation with conjugate}

A general linear quaternionic equation containing the conjugate of the quaternionic indeterminate $\mathbf{q}$ is of the following form:

$$
\sum_{r=1}^{\alpha} \mathbf{c}_{r} \mathbf{q} \mathbf{b}_{r}-\sum_{r=\alpha+1}^{n-1} \mathbf{c}_{r} \overline{\mathbf{q}} \mathbf{b}_{r}=\mathbf{d},
$$

where $0 \leq \alpha<n$, and the $\mathbf{c}_{r}, \mathbf{b}_{r}, \mathbf{d}$ are given quaternions.

Let $\mathbf{q}=x_{0}+\mathbf{x}$, where $x_{0}, \mathbf{x}$ are the real part and the pure imaginary part of $\mathbf{q}$ respectively. Set

$$
\mathbf{h}:=\sum_{r=1}^{\alpha} \mathbf{c}_{r} \mathbf{b}_{r}-\sum_{r=\alpha+1}^{n-1} \mathbf{c}_{r} \mathbf{b}_{r} .
$$


Then (6.1) can be written as

$$
\sum_{r=1}^{n-1} \mathbf{c}_{r} \mathbf{x b}_{r}=\mathbf{d}-x_{0} \mathbf{h} .
$$

Applying Theorem 4.3 to equation (6.3), then using the notation of (5.16), we get

$$
\Delta \mathbf{x}=\Phi \mathbf{d}-x_{0} \Phi \mathbf{h} .
$$

By $\operatorname{Re}(\mathbf{x})=0$, we have $\operatorname{Re}(\Phi \mathbf{d})-x_{0} \operatorname{Re}(\Phi \mathbf{h})=0$, so

$$
x_{0}=\frac{\operatorname{Re}(\Phi \mathbf{d})}{\operatorname{Re}(\Phi \mathbf{h})} .
$$

Lemma 6.1. Let $\mathbf{M}$ be the coefficient matrix of the associated real linear system of (6.1), then

$$
\operatorname{det}(\mathbf{M})=-\frac{1}{3} \operatorname{Re}(\Phi \mathbf{h})
$$

Proof. By definition,

$$
\mathbf{M}(1, \mathbf{i}, \mathbf{j}, \mathbf{k})=(\mathbf{h}, \mathbf{A} \mathbf{i}, \mathbf{A} \mathbf{j}, \mathbf{A k}),
$$

where $\mathbf{A}$ is the coefficient matrix of the associated real linear equations of (1.1). When $\mathbf{A}$ is invertible,

$$
\mathbf{A}^{-1} \mathbf{M}(1, \mathbf{i}, \mathbf{j}, \mathbf{k})=\left(\mathbf{A}^{-1} \mathbf{h}, \mathbf{i}, \mathbf{j}, \mathbf{k}\right)=\left(\operatorname{Re}\left(\mathbf{A}^{-1} \mathbf{h}\right)+\operatorname{Im}\left(\mathbf{A}^{-1} \mathbf{h}\right), \mathbf{i}, \mathbf{j}, \mathbf{k}\right),
$$

SO

$$
\begin{aligned}
\operatorname{det}\left(\mathbf{A}^{-1} \mathbf{M}\right) & =(\operatorname{det}(\mathbf{A}))^{-1} \operatorname{det}(\mathbf{M}) \\
& =\operatorname{Re}\left(\mathbf{A}^{-1} \mathbf{h}\right) \\
& =(\operatorname{det}(\mathbf{A}))^{-1} \operatorname{Re}(\operatorname{adj}(\mathbf{A}) \mathbf{h}) \\
& =-\frac{1}{3}(\operatorname{det}(\mathbf{A}))^{-1} \operatorname{Re}(\Phi \mathbf{h}) .
\end{aligned}
$$

When $\mathbf{A}$ is not invertible, 6.6 is still valid by the continuity of both sides of it in the coordinates of the quaternionic coefficients of (6.1).

The following result is direct from (6.4), (6.5) and (6.6):

Theorem 6.2. When $\operatorname{det}(\mathbf{A})=-\Delta / 3 \neq 0$ and $\operatorname{det}(\mathbf{M})=-\operatorname{Re}(\Phi \mathbf{h}) / 3 \neq 0$, the solution to 6.1) is

$$
\Delta \operatorname{Re}(\Phi \mathbf{h}) \mathbf{q}=\Delta \operatorname{Re}(\Phi \mathbf{d})-\operatorname{Re}(\Phi \mathbf{d}) \Phi \mathbf{h}+\operatorname{Re}(\Phi \mathbf{h}) \Phi \mathbf{d} .
$$

\section{References}

[1] S. De Leo and P. Rotelli. The quaternionic Dirac Lagrangian. Mod. Phys. Lett. A 11 (5), 357-366. 1996.

[2] S. Eilenberg and I. Niven. The "fundamental theorem of algebra" for quaternions. Bull. Amer. Math. Soc. 50: 246-248, 1944.

[3] L. van Elfrinkhof. Eene eigenschap van de orthogonale substitutie van de vierde orde. Handelingen Van Het Nederlandsch Natuurkundig En Geneeskundig Congres, Delft, pp. 237-240, 1897. 
[4] D. Hestenes and G. Sobczyk. Clifford Algebras to Geometric Calculus. D. Reidel, Dordrecht, 1984.

[5] J. Helmstetter. The Quaternionic Equation $a x+x b=c$, Adv. Appl. Clifford Algebras 22: 1055-1059, 2012.

[6] D. Janovská and G. Opfer. Linear equations in quaternions. Numerical Mathematics and Advanced Applications, Proceedings of ENUMATH 2005, A. B. Castro, D. Gómez, P. Quintela, P. Saldago (eds.), Springer, Berlin, Heidelberg, New York, pp. 945-953, 2006.

[7] D. Janovská and G. Opfer. Linear equations in quaternionic variables. Mitt. Math. Ges. Hamburg 27: 223-234, 2008.

[8] D. Janovská and G. Opfer. A note on the computation of all zeros of simple quaternionic polynomial. SIAM J. Numerical Analysis 48: 244-256, 2010.

[9] D. Janovská and G. Opfer. The classification and the computation of the zeros of quaternionic, two-sided polynomials. Numer. Math. 115: 81-100, 2010.

[10] R.E. Johnson. On the equation $\chi \alpha=\gamma \chi+\beta$ over an algebraic division ring. Bull. Amer. Math. Soc. 50: 202-207, 1944.

[11] B. Kalantari. Algorithms for quaternion polynomial root-finding. Journal of Complexity 29: 302-322, 2013.

[12] H. Li. Geometric Reasoning with Invariant Algebras. World Scientific, Singapore, 2008.

[13] I. Niven. Equations in quaternions. Amer. Math. Monthly 48: 654-661, 1941.

[14] A. Pogorui and M. Shapiro. On the structure of the set of zeros of quaternionic polynomials. Complex Var. Elliptic Funct. 49: 379-389, 2004.

[15] R.M. Porter. Quaternionic linear and quadratic equations. J. Natur. Geom. 11: 101-106, 1997.

[16] L. Rodman. Topics in Quaternion Linear Algebra. Princeton University Press, Princeton, 2014.

[17] R. Serôdio, E. Pereira, and J. Vitória. Computing the zeros of quaternionic polynomials, Comput. Math. Appl. 42: 1229-1237. 2001.

[18] V.S. Shpakivskyi. Linear Quaternionic Equations and Their Systems, Adv. Appl. Clifford Algebras 21: 637-645, 2011.

[19] C. Schwartz. Linear equations for noncommutative algebras. Available: http://socrates.berkeley.edu/ ${ }^{\sim}$ schwrtz/Linear.pdf, 2013.

[20] J.J. Sylvester. Sur l'equations en matrices $p x=x q$, C.R. Acad. Sci. Paris. 99(2): 67-71, 115-116, 1884.

[21] J.D. Turner. Solving linear and quadratic quaternion equations. Journal of Guidance, Control, and Dynamics, 29(6): 1420-1423, 2006.

Changpeng Shao, Hongbo Li, Lei Huang

KLMM, AMSS and UCAS

Chinese Academy of Sciences

Beijing 100190, China

e-mail:

shaochangpeng11@mails.ucas.ac.cn,

hli@mmrc.iss.ac.cn,

lhuang@mmrc.iss.ac.cn 